\title{
NUEVOS DATOS SOBRE LA PRODUCCIÓN DE CERÁMICA DE COCINA Y DE LOZA BASTA DE SEVILLA EN LOS SIGLOS XV-XVI
}

\author{
NEW DATA ON COOKING WARE AND GLAZED COARSE POTTERY \\ PRODUCTION IN SEVILLE DURING THE $15^{\text {TH }}$ AND $16^{\text {TH }}$ CENTURIES
}

\author{
CRISTINA FERNÁNDEZ DE MARCOS GARCÍA* / JAUME BUXEDA I GARRIGÓS** / \\ FERNANDO AMORES $* * *$
}

\begin{abstract}
Resumen: A partir de la llegada de los españoles a América, la ciudad de Sevilla se convirtió en el mayor centro productor cerámico de Europa gracias a su elección como único puerto comercial del monopolio que la Corona castellana estableció con el "Nuevo Mundo", por medio de la Casa de Contratación. La producción sevillana se ha estudiado ampliamente desde el punto de vista arqueológico y decorativo, pero actualmente aún son escasos los trabajos arqueométricos al respecto. Por ello, y con el objetivo de obtener una visión adecuada de la gran complejidad e importancia de la producción cerámica de Sevilla, se han caracterizado arqueométricamente un total de 34 muestras procedentes de contextos arqueológicos sevillanos datados entre finales del siglo XV y el siglo XVI. Estas muestras corresponden a cerámica de cocina y cerámica común vidriada, clases cerámicas que casi no se han tratado en investigaciones anteriores que se habían centrado principalmente en la mayólica y en los contenedores de transporte. Los análisis se han realizado mediante las técnicas de fluorescencia de rayos $\mathrm{X}$ (FRX) y difracción de rayos $\mathrm{X}(\mathrm{DRX}) \mathrm{y}$ han permitido establecer dos nuevos grupos de referencia, así como obtener una primera visión sobre la técnica de producción de dichas cerámicas.
\end{abstract}

\footnotetext{
* Cultura Material i Arqueometria UB (ARQUB, GRACPE), Departament d'Història i Arqueologia, Universitat de Barcelona, c/ de Montalegre, 6, 08001 Barcelona. Correo-e: cfernandezdemarcos@ub.edu.
}

\begin{abstract}
After the arrival of the Spaniards to America, Seville was chosen to host the Casa de Contratación, the central trading house responsible for the intended monopoly that should control all the relations with the new territories. Consequently, Seville became one of the main ceramic production centers of Europe. Because of its relevance, the pottery production of Seville has received an important attention from the historical of art and archaeological point of view, but the archaeometric research is still scarce. Therefore, in order to achieve a better understanding of this production center, 34 vessels unearthed in Seville have been analyzed. These ceramics correspond to late $15^{\text {th }}$ and $16^{\text {th }}$ century glazed coarse and cooking wares, ceramic classes that have been rarely considered in previous studies centered on majolica and transport jars. The samples have been characterized by means of X-ray fluorescence (XRF) and X-ray diffraction (XRD). The results have enabled to identify two new reference groups as well as their technique of manufacture.
\end{abstract}

** Cultura Material i Arqueometria UB (ARQUB, GRACPE), Departament d'Història i Arqueologia, Universitat de Barcelona, c/ de Montalegre,6, 08001 Barcelona. Correo-e: jbuxeda@ub.edu.

*** Departamento de Prehistoria y Arqueología, Universidad de Sevilla, C/ Doña María de Padilla, s/no, 41004 Sevilla. Correo-e: famores@us.es. 
Palabras clave: Loza basta vidriada, cerámica de cocina vidriada, Sevilla, fluorescencia de rayos X, difracción de rayos $\mathrm{X}$, proveniencia, técnica.

\section{INTRODUCCIÓN}

La llegada de la corona de Castilla a América a finales del siglo XV tendría una fuerte repercusión en todos los ámbitos para la ciudad de Sevilla al ser escogida, por orden de los Reyes Católicos, como único puerto desde el que se establecería un monopolio comercial con el "Nuevo Mundo". Así, todos los barcos que querían comerciar con América, no solamente los de la monarquía hispánica, sino también los de toda Europa deberían pasar por Sevilla, convirtiendo la ciudad en "la puerta de las Indias". El motivo principal para esta elección fue su situación estratégica al estar resguardada de los ataques piratas de la costa pero sin estar lejos de ella; además, el puerto ya había empezado a desarrollar unas estructuras sólidas previamente a la llegada a América dado que ya existían relaciones comerciales con ciudades italianas, de Flandes, de Inglaterra y del norte de Europa, las cuales, aunque con menor importancia, se mantuvieron a lo largo de los siglos XVI y XVII (Chaunu 1983: 24).

Esta situación de centro del monopolio con América se prolongó desde 1503, año en que se creó la Casa de la Contratación, hasta 1679-80 cuando la ciudad de Cádiz cogió el relevo como puerto principal del comercio atlántico (Martínez 1993: 109, Elliott 2005: 193). La Casa de la Contratación tenía la finalidad de proteger los territorios del "Nuevo Mundo" a nivel económico, ya que esos territorios tenían que ser una fuente inagotable de riqueza. De este modo, desde el inicio de la Carrera de Indias, la Casa de la Contratación gestionó todo lo que tenía relación con el comercio con América haciendo de aduana, inspeccionando todas las naves y registrando tanto las mercancías como las personas que viajaban en las flotas.

En este marco en que la ciudad debía abastecer el "Nuevo Mundo" de todo tipo de productos, Sevilla tuvo un gran crecimiento convirtiéndose en un gran centro económico a nivel europeo (Mena 1998). Con ello, la producción cerámica de la ciudad se convirtió en una de las actividades más significativas, ya que se exportaba en grandes cantidades hacia América. Este aumento de la producción cerámica también se acompañó con la mejora de las técnicas de fabricación. Como consecuencia, Sevilla se convirtió en el
Keywords: Glazed coarse ware, glazed cooking ware, Seville, $\mathrm{X}$-ray fluorescence, $\mathrm{X}$-ray diffraction, provenance, technique.

referente de la elaboración de cerámica en la península ibérica, Europa y América y muchos ceramistas europeos, como el italiano Niculoso Pisano, se trasladaron a la ciudad, estableciendo allí su taller. Numerosos talleres se situaron extramuros en el barrio de Triana hasta albergar, durante el siglo XVI, unos 50, el $80 \%$ de los talleres de Sevilla. El resto de talleres se repartían intramuros entre las collaciones de San Pedro, San Vicente y San Marcos, pero también en el arrabal de San Telmo (fig. 1). A lo largo del siglo XVI, el barrio de Triana tuvo un crecimiento económico acelerado; los ceramistas aquí situados se beneficiaban de su ubicación cercana al río, una vía de comunicación rápida y barata que aprovechaban para el transporte de las materias primas (Morales 1989: 153, Sánchez 1994: 75).

Cabe destacar, sin embargo, que esta industria cerámica sevillana se ha estudiado ampliamente, pero de modo desigual. La llamada loza fina o mayólica, por su carácter decorativo, siempre ha tenido un mayor protagonismo en las investigaciones (Pleguezuelo 1997, 2003a, 2003b), dejando de lado la loza basta, mucho menos estudiada. En este sentido, los primeros trabajos se centraron especialmente en la mayólica desde el punto de vista de la historia del arte, haciendo énfasis en el estilo de las decoraciones como indicador cronológico. También en América los estudios arqueológicos y arqueométricos sobre contextos de los siglos XVIXVII se centraron en la mayólica de proveniencia, $a$ priori, sevillana, dejando a un lado las producciones locales (distinguimos, por convención, procedencia, como lugar de encuentro o de recuperación de un objeto, de proveniencia, como lugar de origen de ese objeto o de la materia prima de ese objeto. Vid. Buxeda 2001). Entre estos trabajos destacan los de Goggin (1968), Lister y Lister $(1974,1982,1987)$ y Deagan (1987). Asimismo, fueron también trabajos americanos los primeros en abordar la caracterización arqueométrica de las cerámicas de Sevilla, tanto para poder distinguir las cerámicas exportadas de la península ibérica hacia América, como para poder identificar la producción americana posterior. Estos estudios se realizaron básicamente mediante la técnica de Análisis por Activación Neutrónica (AAN) (Olin et al. 1978, Olin y Blackman 1989, Olin y Myers 1992, Maggetti 1986, Maggetti et al. 1984, Myers et al. 1992, Jamieson y 


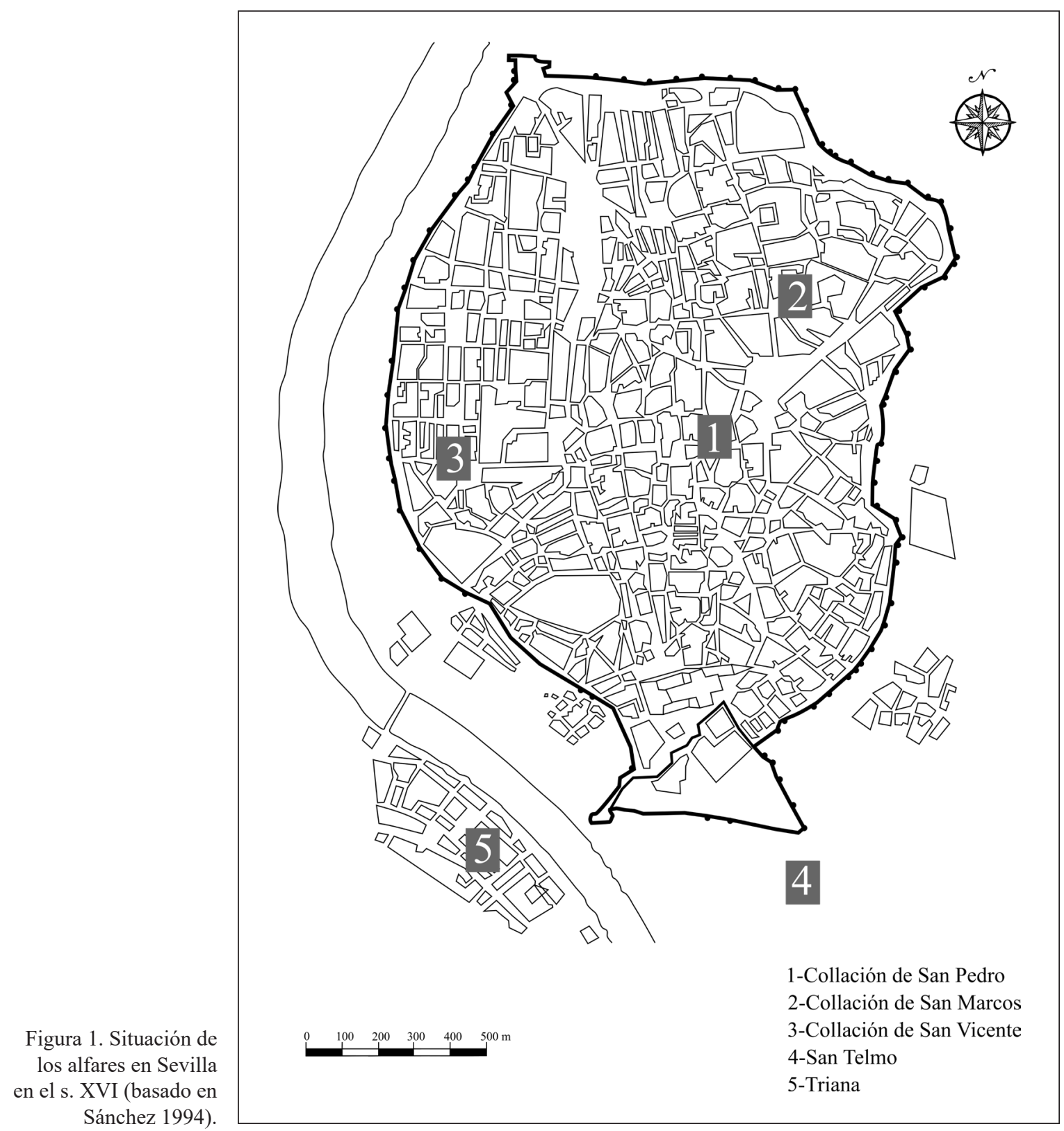

Hancock 2004). Posteriormente se realizaron también, mediante AAN, análisis sobre mayólicas y cerámicas vidriadas provenientes de México (Blackman et al. 2006, Fournier y Blackman 2008).

En los últimos años, los estudios respecto a la cerámica sevillana se han incrementado de manera cualitativa. Se han desarrollado avances de forma paralela en la investigación documental y arqueológica, que, más recientemente, han llevado a aumentar los análisis arqueométricos. En este contexto, desde el punto de vista documental, destaca la obra de Sánchez (1994,
1996, 1998) que ha realizado estudios sobre el funcionamiento de la producción cerámica en la ciudad, sobre el funcionamiento del gremio de ceramistas y sobre el registro de la cerámica sevillana exportada hacia América a través de la documentación del Archivo General de Indias.

Sánchez (1994: 43-49) también trabajó la información documental acerca de las zonas de extracción de arcilla en el siglo XVI. Los "barreros" referenciados se situaban en la cornisa del Aljarafe, en la Isleta de la Cartuja, en la dehesa de Tablada y en el campo de 


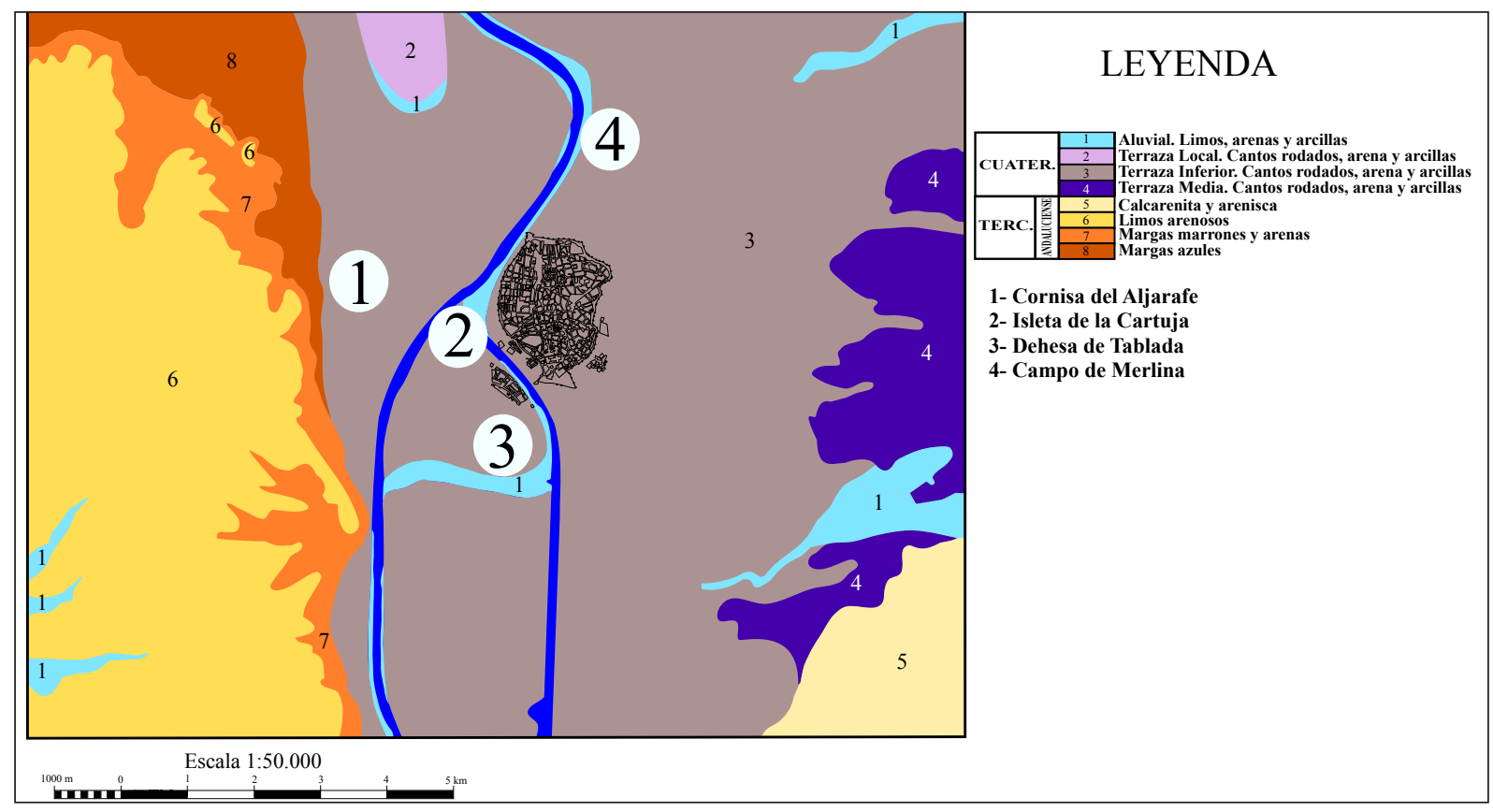

Figura 2. Mapa geológico de Sevilla basado en el Mapa geológico de España E. 1:50.000, hoja 984, Sevilla (IGME 1975), con indicación de las zonas de extracción de arcilla según Sánchez (1994).

Merlina. Cabe destacar que, la gran importancia que tuvo Sevilla como centro productor de cerámica no se podría entender sin el contexto geológico sobre el que está asentada la ciudad. Sin lugar a dudas, la ubicación tan próxima de la materia prima facilitó y aceleró el trabajo de los alfareros, además de reducir los costes de transporte (fig. 2).

A este corpus documental, se le añade un conocimiento arqueológico basado especialmente en las excavaciones que desde 1984 se han llevado a cabo en un gran número de solares con contextos de época moderna. A pesar de ello, también cabe decir que su estudio es aún, en algunos casos, insuficiente o, en otros casos, inexistente. Este problema se puede considerar más grave si se tiene en cuenta la pérdida de información sobre contextos excavados sin control entre los años 1960 y 1980 (Amores y López 2009: 569). Aun así, en el barrio de Triana se han recuperado diversos restos de alfares y hornos que cronológicamente se enmarcan entre los siglos XIII y XIX. Entre las excavaciones más importantes destacan las de La Cartuja (1987-1992) (Amores et al. 1997), el número 44 de la calle Pureza (1986-1987) (Lorenzo et al. 1990), en donde se recuperaron los restos de un obrador de cerámica atribuido a Niculoso Pisano, la Plaza de Armas (1997-1998) (Mercado et al. 2001), con los posibles deshechos del taller de Tomás Pesaro, el Castillo de San
Jorge (1998-2000) (Hunt 2001) y las excavaciones del Monasterio de San Clemente, en donde aparecieron varios rellenos de cerámica en la estructura arquitectónica del conjunto, en los cuales se encontraron piezas trianeras y piezas de tradición talaverana, así como importaciones procedentes de Italia, Lisboa e incluso China (Ojeda 1989; Tabales 1991). Así, en los últimos años, se han podido realizar estudios arqueométricos del material recuperado en varias de dichas excavaciones, como el de Polvorinos del Río y Castaing (2010), pero también se han realizado recientes análisis sobre las cerámicas del pecio de Tortugas (Florida, EUA) identificado con el Buen Jesús y Nuestra Señora del Rosario hundido en 1622 (Hughes 2014). Con todo, cabe destacar el trabajo de Iñañez (2007), en el que se caracterizaron una serie de muestras de mayólica sevillana, la mayoría procedentes del yacimiento de Plaza de Armas, junto con la de otros centros productores peninsulares. Previamente, en 2003, se había realizado un estudio sobre mayólicas de los centros de Talavera de la Reina y Triana, en el que se pudieron discernir químicamente las dos producciones (Buxeda et al. 2003). Estos últimos estudios han servido de base para el actual proyecto Tecnolonial, en el cual se incluye el presente trabajo. Gracias a este proyecto se está dando un nuevo impulso al conocimiento de la cerámica producida en Sevilla considerándose no sólo mayólicas, sino 
también cerámicas de transporte y almacenamiento (Ferrer et al. 2013, 2015a, 2015b; Buxeda et al. 2015), así como las cerámicas de cocina y vidriada común tratadas en el presente artículo.

\section{MUESTREO Y RUTINA ANALÍTICA}

En el presente estudio se ha considerado una muestra de 34 individuos pertenecientes a las series de cerámica vidriada doméstica, de mesa y cocina menos estudiadas hasta el presente, además de un cangilón perteneciente a la llamada cerámica agrícola-industrial (Tabla1). Los individuos analizadas han sido clasificados según la tipología realizada por Amores y Chisvert (1993) (Fig. 3), establecida para esta cerámica común sevillana de los siglos XV y XVI a partir de los hallazgos realizados en los rellenos de bóvedas de edificios como la Catedral de Sevilla o el Monasterio de la Cartuja. En estos rellenos, y con el propósito de aligerar las estructuras arquitectónicas, se emplearon diversas piezas de loza basta que habían sufrido defectos de fabricación. La mayoría de las bóvedas estudiadas, como las del claustro de monjes, las de la capilla de Santa Catalina o las de la Sala Capitular, pertenecían al monasterio de la Cartuja, aunque también se estudiaron los materiales procedentes de las excavaciones de las bóvedas de la capilla de la Virgen de la Antigua en la Catedral de Sevilla. Además, el estudio se complementó con los materiales del monasterio de San Isidoro del Campo, en Santiponce, y otros materiales procedentes de Marchena y de Carmona (Amores y Chisvert 1993: 271).

Los individuos aquí analizados se sitúan cronológicamente en los siglos XV-XVI y proceden de las excavaciones realizadas en el Castillo de San Jorge, en el monasterio de La Cartuja de Sevilla, en el número 104 de la calle Pureza y en una de las bóvedas de la capilla de San Isidoro de la Catedral de Sevilla (Tabla 1). La mayoría de las piezas analizadas provienen de las intervenciones en el Castillo de San Jorge, que fue sede de la Inquisición desde 1481 hasta 1785. Las campañas de excavación se realizaron de forma continua entre 1998 y 2000, documentándose fases desde época tardorromana hasta el siglo XIX (Hunt 2001). Respecto al monasterio de La Cartuja, las intervenciones se realizaron entre 1987 y 1992 con motivo de la Exposición Universal de Sevilla de 1992 (Amores et al. 1997; Amores 1998, 1999). En estas campañas de excavación se documentaron las dos etapas más importantes de desarrollo del recinto: la del monasterio cartujo (ss. XV-XIX) y la de la fábrica de lozas (ss. XIX-XX). En relación a las intervenciones en la

Tabla 1. Tipología y procedencia de los individuos muestreados.

\begin{tabular}{|c|c|c|c|c|c|c|}
\hline \multirow[b]{2}{*}{ Grupo } & \multirow[b]{2}{*}{ Forma } & \multicolumn{4}{|c|}{ Muestras } & \multirow[b]{2}{*}{ Total } \\
\hline & & $\begin{array}{l}\text { Bóveda de } \\
\text { San Isidoro }\end{array}$ & La Cartuja & C/ Pureza, 104 & $\begin{array}{l}\text { Castillo de } \\
\text { San Jorge }\end{array}$ & \\
\hline \multirow{2}{*}{$\begin{array}{l}\text { Doméstico } \\
\text { General }\end{array}$} & Lebrillo & & SEV068, 70 & SEV071 & SEV077,79,85,89,93,94 & 9 \\
\hline & Bacín & SEV063, 64 & & SEV072 & & 3 \\
\hline \multirow{4}{*}{ Mesa } & Jarro & & SEV066 & & SEV081,92 & 3 \\
\hline & Jarra & & & & SEV075,83,96 & 3 \\
\hline & Jarrito & & SEV069 & & SEV074,84,87,95 & 5 \\
\hline & Jarrita & & & & SEV073,86 & 2 \\
\hline \multirow{3}{*}{ Cocina } & Cazuela & & & & SEV076,78,80,82,90,91 & 6 \\
\hline & Olla & & SEV067 & & & 1 \\
\hline & Informe & & & & SEV088 & 1 \\
\hline \multirow[t]{2}{*}{$\begin{array}{l}\text { Agrícola- } \\
\text { Industrial }\end{array}$} & Cangilón & SEV065 & & & & 1 \\
\hline & Total & 3 & 5 & 2 & 24 & 34 \\
\hline
\end{tabular}




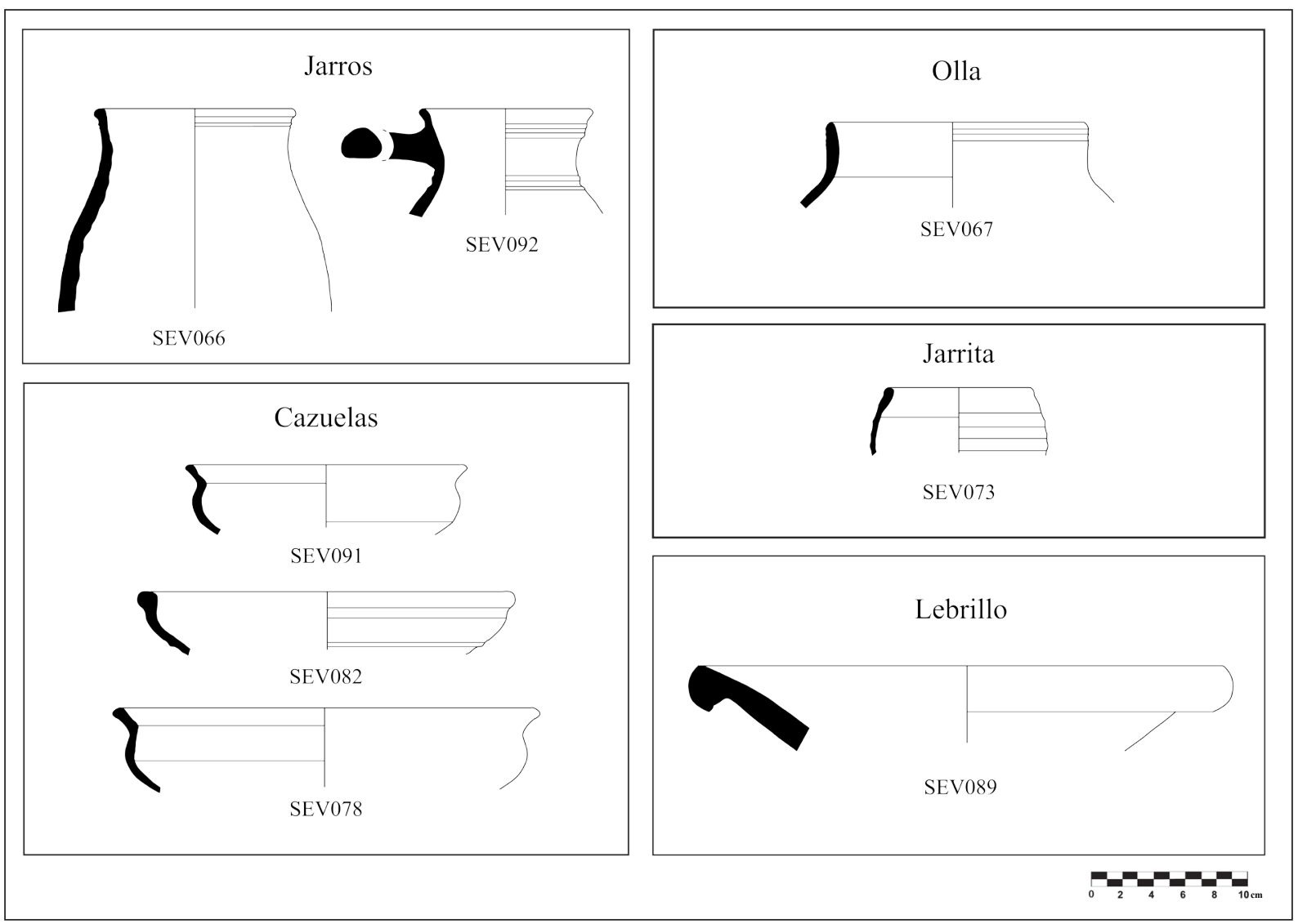

Figura 3. Ejemplos de algunas de las piezas analizadas.

bóveda de San Isidoro y en el número 104 de la calle Pureza, actualmente continúan inéditas.

Los 34 individuos han sido caracterizados químicamente por fluorescencia de rayos X (FRX) y mineralógicamente por difracción de rayos X (DRX). Para su caracterización química y mineralógica, de cada cerámica se ha tomado una muestra de unos $15 \mathrm{~g}$, cuya superficie fue eliminada mecánicamente. Posteriormente, las muestras fueron molturadas y homogeneizadas mediante un molino de bolas con celda de carburo de tungsteno Spex Mixer mod. 8000. La caracterización por FRX se ha realizado a partir del polvo previamente secado en una estufa a $105^{\circ} \mathrm{C}$ durante $12 \mathrm{~h}$. Para la determinación de los elementos mayores y menores, se han hecho preparaciones de duplicados de perlas de 30 $\mathrm{mm}$ de diámetro, usando $0.3 \mathrm{~g}$ de espécimen en fusión alcalina con $5.7 \mathrm{~g}$ de tetraborato de litio $\left(\mathrm{Li}_{2} \mathrm{~B}_{4} \mathrm{O}_{7}\right)$ en una dilución $1 / 20$. A esta mezcla se le añaden $5 \mathrm{mg}$ de ioduro de litio (LiI) para controlar la tensión superficial de la perla. La mezcla final se deposita en un crisol de $\mathrm{Pt}-\mathrm{Au}$ (95:5) para su fusión en un horno de inducción de alta frecuencia Perl'X-3 de PANalytical a una temperatura máxima de $1125^{\circ} \mathrm{C}$. Para la determinación de los elementos traza, se han realizado preparaciones de pastillas a partir del espécimen anteriormente preparado, seco y de tamaño de grano $<80 \mu \mathrm{m}$, mezclado con $2 \mathrm{ml}$ de una solución de resina sintética n-butil metacrilato (Elvacite 2044, en $20 \%$ de acetona). Esta mezcla, homogeneizada manualmente en un mortero de ágata hasta sequedad y dispuesta sobre una base de ácido bórico $\left(\mathrm{H}_{3} \mathrm{BO}_{3}\right)$ en un recipiente de aluminio de $40 \mathrm{~mm}$ de diámetro, se somete a una presión de $200 \mathrm{kN}$ durante $60 \mathrm{~s}$ en una prensa Herzog. La cuantificación se ha efectuado con un espectrómetro Axios ${ }^{m A X}$-Advanced PANalyical con fuente de excitación de Rh utilizando una recta de calibración configurada con 56 patrones (Estándares Geológicos Internacionales). Las interferencias han sido tomadas en consideración y los efectos matriz han sido corregidos usando el software para elementos traza PANalyical Pro-Trace. Así, se han determinado los elementos: $\mathrm{Na}_{2} \mathrm{O}, \mathrm{MgO}, \mathrm{Al}_{2} \mathrm{O}_{3}, \mathrm{SiO}_{2}, \mathrm{P}_{2} \mathrm{O}_{5}$, $\mathrm{K}_{2} \mathrm{O}, \mathrm{CaO}, \mathrm{TiO}_{2}, \mathrm{~V}, \mathrm{Cr}, \mathrm{MnO}, \mathrm{Fe}_{2} \mathrm{O}_{3}$ (como Fe total), 
Co, Ni, Cu, Zn, Ga, Rb, Sr, Y, Zr, Nb, Mo, Sn, Ba, Ce, $\mathrm{W}, \mathrm{Pb}$ y Th. Finalmente, también se ha calculado la pérdida al fuego (PAF) a partir de calcinaciones de $0.3 \mathrm{~g}$ de espécimen seco a $950^{\circ} \mathrm{C}$ durante $3 \mathrm{~h}$. Las calcinaciones se han efectuado en una mufla Heraeus mod. M-110, usando una rampa de calentamiento de $3.4^{\circ} \mathrm{C} \mathrm{min}^{-1} \mathrm{y}$ enfriado libre. La suma de las concentraciones determinadas y la pérdida al fuego se encuentran en el rango (99-100)\%. Una detallada descripción de las condiciones analíticas, la precisión y la exactitud ha sido publicada anteriormente (Hein et al. 2002).

La composición mineralógica de estos individuos ha sido estudiada mediante la DRX utilizando el polvo de los especímenes previamente preparados. Así, se ha usado un difractómetro de geometría BraggBrentano PANalytical X'Pert PRO MPD Alpha-1 (radio $=240 \mathrm{~mm})$, trabajando con la radiación $\mathrm{K} \alpha$ del $\mathrm{Cu}$ $(\lambda=1.5418 \AA)$, con un monocromador primario focalizador de Ge (111) y una potencia de trabajo de $1.8 \mathrm{~kW}$ (45 kV-40 mA). El difractómetro cuenta con una ventana de divergencia variable para conseguir una longitud irradiada de dirección paralela al haz de rayos $\mathrm{X}$ de $10 \mathrm{~mm}$, con máscara en el haz incidente que regula la longitud irradiada sobre la muestra en la dirección axial a $12 \mathrm{~mm}$, así como un detector X'Celerator con longitud activa de $3.347^{\circ}$. Las mediciones han sido realizadas de $(5 \text { a } 80)^{\circ} 2 \theta$ con una medida de paso de $0.026^{\circ}$ y un tiempo de conteo de $50 \mathrm{~s}$. Las fases cristalinas presentes en cada muestra analizada han sido identificadas y evaluadas con el paquete de programas X'Pert HighScore Plus de PANalytical que incluye el banco de datos del International Centre for Diffraction DataJoint Committee of Powder Diffraction Standards, 2006 (ICDD-JCPDS).

\section{RESULTADOS ARQUEOMÉTRICOS}

\subsection{Caracterización química}

Los resultados del análisis químico realizado por FRX, es decir las concentraciones elementales determinadas, corresponden a un caso especial del espacio proyectivo $\mathrm{d}+1$-dimensional de puntos proyectivos desde el origen que son proyectados en el símplex $S^{d}$. Así, los puntos proyectivos están representados por coordenadas homogéneas que tienen una suma constante $k\left(k \in R_{+}\right)$,

$$
\begin{gathered}
\mathbf{x}=\left[\mathrm{x}_{1}, \ldots, \mathrm{x}_{\mathrm{d}}, \mathrm{x}_{d+1}\right] \mid \mathrm{x}_{\mathrm{i}} \geq 0(\mathrm{i}=1, \ldots, \mathrm{d}, \mathrm{d}+1), \mathrm{x}_{1}+\ldots \\
+\mathrm{x}_{\mathrm{d}}+\mathrm{x}_{d+1}=k,
\end{gathered}
$$

(en este caso, $\mathrm{k}=100$ ). El espacio vectorial de los puntos proyectivos es el ortante positivo $R_{+}^{d+1}$ y estos puntos proyectivos y sus proyecciones en el símplex siguen un modelo multiplicativo con una métrica de intervalos logarítmicos (Barceló-Vidal et al. 2001; Aitchison 2005; Buxeda 2008). Debido a ello, para su tratamiento estadístico, los datos obtenidos han sido transformados utilizando la transformación alr en logaritmos de razones, según

$$
\mathbf{x} \in S^{\mathrm{d}} \rightarrow \mathbf{y}=\log \left(\frac{\mathbf{x}_{d}}{\mathbf{x}_{\mathrm{d}+1}}\right) \in R^{\mathrm{d}} \quad \text { (ecuación 1) }
$$

donde $S^{d}$ es el símplex $d$-dimensional, $\mathbf{x}_{\mathrm{d}}=\left[\mathrm{x}_{1}, \ldots, \mathrm{x}_{\mathrm{d}}\right]$, o la transformación clr en logaritmos de razones centradas según

$$
\mathbf{x} \in \mathrm{S}^{d} \rightarrow \mathbf{z}=\log \left(\frac{\mathbf{x}}{g(\mathbf{x})}\right) \in \mathrm{R}^{d+1} \quad \text { (ecuación 2) }
$$

donde $S^{d}$ es el símplex $d$-dimensional y g(x) es la media geométrica de todos los $\mathrm{d}+1$ componentes de $\mathbf{x}$ (Aitchison 1986; Buxeda 1999).

En el desarrollo del tratamiento estadístico, los componentes $\mathrm{W}$ y Co no se pueden considerar por las posibles contaminaciones derivadas del uso del molino de bolas equipado con una celda de carburo de tungsteno, empleado durante el proceso de pulverización y homogenización de las muestras. Igualmente, Mo y Sn también han sido excluidos porque normalmente en la cerámica presentan concentraciones muy bajas, fuera de los límites de regresión, ocasionando indeterminaciones e imprecisiones analíticas. Además, en este trabajo hace falta prescindir de otros elementos porque las cerámicas analizadas presentan vidriados de $\mathrm{Pb}$, cuya difusión en la matriz provoca interferencias a otros elementos que no pueden ser corregidas óptimamente. Este es el caso del Rb, el Y y el Ga, así como del Th, aunque a un nivel menor (Iñañez 2007: 146-147). Finalmente, los elementos $\mathrm{P}_{2} \mathrm{O}_{5}$ y Cu pueden verse alterados muy fácilmente por procesos postdeposicionales $\mathrm{o}$, en el caso del $\mathrm{Cu}$, por los mismos vidriados, por lo que tampoco son tenidos en cuenta en el estudio estadístico. De este modo, los elementos que serán retenidos en el tratamiento estadístico son: $\mathrm{Na}_{2} \mathrm{O}, \mathrm{MgO}, \mathrm{Al}_{2} \mathrm{O}_{3}, \mathrm{SiO}_{2}$, $\mathrm{K}_{2} \mathrm{O}, \mathrm{CaO}, \mathrm{TiO}_{2}, \mathrm{~V}, \mathrm{Cr}, \mathrm{MnO}, \mathrm{Fe}_{2} \mathrm{O}_{3}$ (como Fe total), $\mathrm{Ni}, \mathrm{Zn}, \mathrm{Sr}, \mathrm{Zr}, \mathrm{Nb}$, Ba y Ce. Para el tratamiento estadístico se empleó el lenguaje y software informático R (R Core Team 2014). 


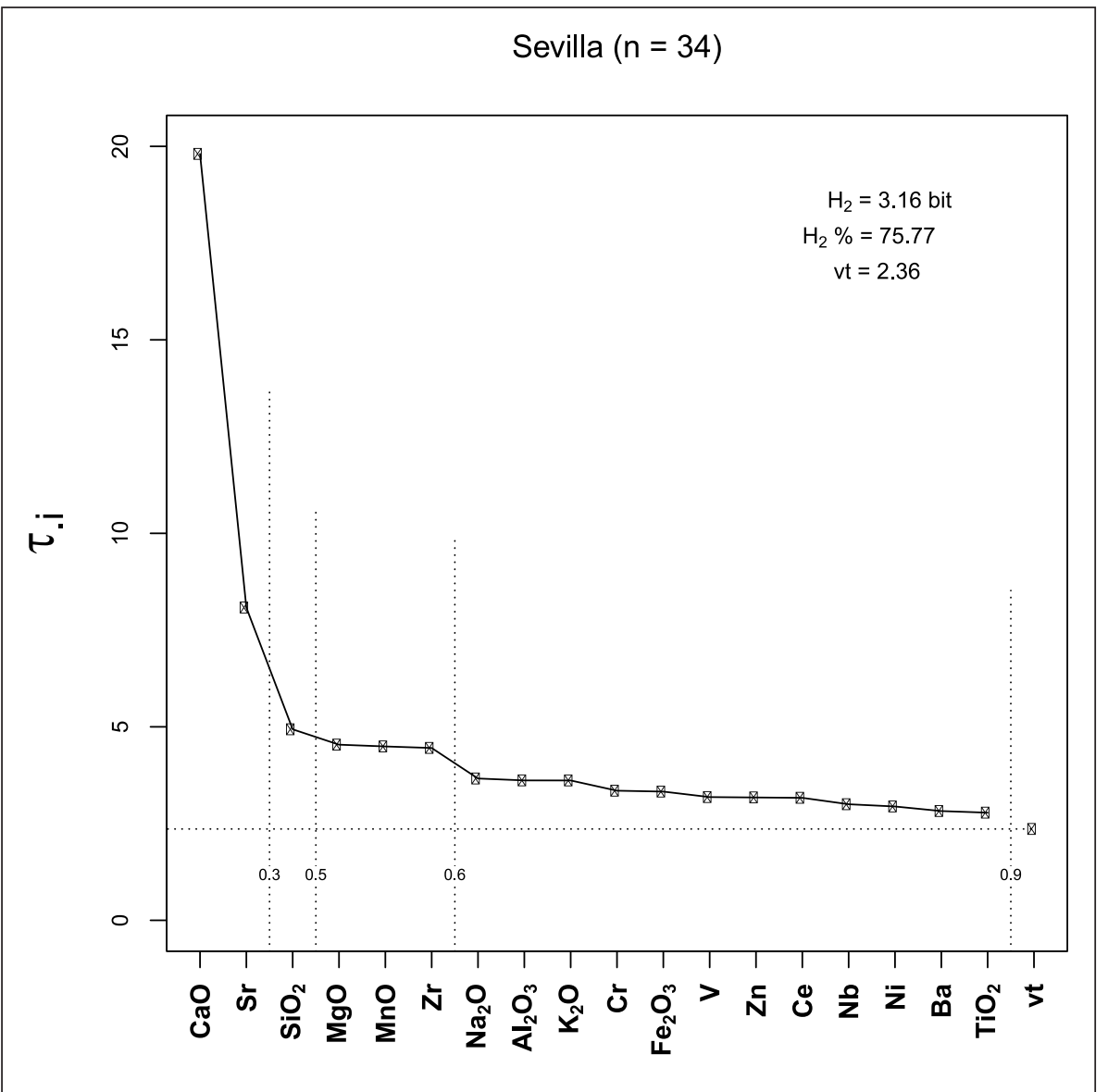

Figura 4. Gráfico de uniformidad composicional de los 34 individuos estudiados, medida con la entropía de la información $\left(\mathrm{H}_{2} \mathrm{y} \mathrm{H}_{2} \%\right.$, su valor relativo) de los componentes retenidos para el análisis estadístico. $\mathrm{vt}=$ variación total.
Para estudiar la variabilidad existente en los datos químicos, calculamos en primer lugar la matriz de variación composicional que permite cuantificar la variación total (vt) presente en la matriz de datos, así como investigar el origen de esta variabilidad. Para los 34 individuos caracterizados, la variación total (vt) es de 2.36, que podemos considerar bastante alta, relacionada, seguramente, con un conjunto poligenético, es decir un conjunto que incluiría materiales de diferentes grupos químicos (Buxeda y Kilikoglou 2003). Así mismo, los elementos que más variabilidad aportan a la muestra son el $\mathrm{CaO}$ y el $\mathrm{Sr}\left(\mathrm{vt} / \tau_{\mathrm{i}}<0.3\right)$, mientras que el $\mathrm{TiO}_{2}$ es el que menos variabilidad aporta (fig. 4). De este modo, para el tratamiento de los datos, el TiO, será utilizado como divisor en la transformación alr en logaritmos de razón (ecuación 1), ya que es el elemento que introduce menos variabilidad.

Una primera apreciación de los datos permite observar claramente una diferenciación entre las cerámicas de cocina y las vidriadas comunes. Para ello se pueden observar los diagramas de dispersión de los componentes $\mathrm{CaO}, \mathrm{Sr}, \mathrm{MnO}, \mathrm{Zr}, \mathrm{Al}_{2} \mathrm{O}_{3}$ y $\mathrm{K}_{2} \mathrm{O}$ con transformación alr utilizando el $\mathrm{TiO}_{2}$ como divisor (fig. 5). Estos componentes corresponden a algunos de los que introducen una mayor variabilidad (fig. 4) y ofrecen una estructura interpretable muy interesante. Es fácil ver cómo las cerámicas de cocina presentan valores relativos bajos especialmente de $\mathrm{CaO}$ y de $\mathrm{Sr}$, a diferencia de lo que ocurre con las cerámicas comunes vidriadas. Ello se observa especialmente bien en los diagramas de estimación de la densidad por kernels de estos componentes transformados, claramente bimodales y con una neta separación entre ambos grupos. Con todo, las cerámicas de cocina presentan una gran variación en los valores relativos de $\mathrm{Al}_{2} \mathrm{O}_{3} \mathrm{y}$ de $\mathrm{Zr}$, que parecen describir una relación inversa entre ellos. Finalmente, se puede apreciar cómo dos de los individuos de cocina presentan valores relativos de $\mathrm{MnO}$ superiores al resto. Por lo que respecta a las cerámicas comunes vidriadas, estas presentan también una gran variación en algunos valores relativos y especialmente en los el $\mathrm{K}_{2} \mathrm{O}$. En este caso, como se verá más adelante, 


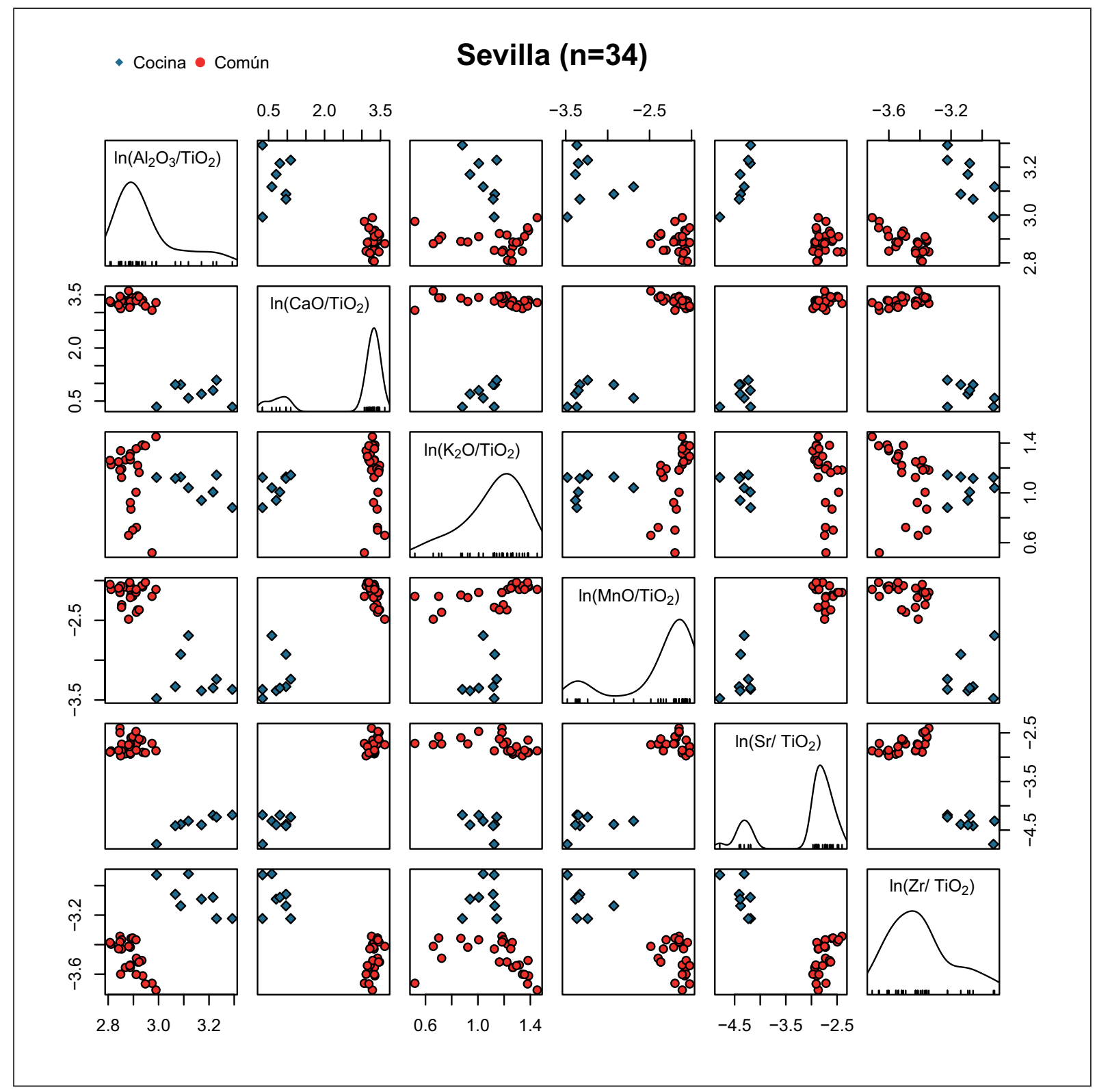

Figura 5. Matriz de diagramas de dispersión de los valores de $\mathrm{Al}_{2} \mathrm{O}_{3}, \mathrm{CaO}, \mathrm{K}_{2} \mathrm{O}, \mathrm{MnO}$, $\mathrm{Sr}$ y Zr en transformación alr utilizando el $\mathrm{TiO}_{2}$ como divisor. En la diagonal, gráficos de estimación de la densidad por kernels de cada uno de los valores transformados.

ello se debe a un doble proceso de alteración que afecta también a los valores relativos del $\mathrm{Na}_{2} \mathrm{O}$. Con todo, este grupo de comunes vidriadas parece ofrecer una diferenciación interna que parece evidente en los valores relativos del $\mathrm{Zr}$ y, en menor medida, del $\mathrm{MnO}$ y el $\mathrm{Al}_{2} \mathrm{O}_{3}$, hecho que se puede observar en los diagramas de dispersión entre estos tres componentes transformados.

Como resumen del estudio estadístico, presentamos el dendrograma resultante del análisis de conglomerados realizado con el algoritmo aglomerativo del centroide y la distancia euclidiana al cuadrado, sobre la subcomposición $\mathrm{Na}_{2} \mathrm{O}, \mathrm{MgO}, \mathrm{Al}_{2} \mathrm{O}_{3}, \mathrm{SiO}_{2}, \mathrm{~K}_{2} \mathrm{O}, \mathrm{CaO}, \mathrm{TiO}_{2}, \mathrm{~V}, \mathrm{Cr}$, $\mathrm{MnO}, \mathrm{Fe}_{2} \mathrm{O}_{3}, \mathrm{Ni}, \mathrm{Zn}$, Sr, $\mathrm{Zr}$, Nb, Ba y Ce, con transformación clr (fig. 6). Aunque los componentes con transformación alr o clr no se corresponden con bases ortonormales en $R^{d}$, sí que la transformación CLR define una estructura métrica en el simplex en la cual el producto interior y la norma, y la distancia que se le 


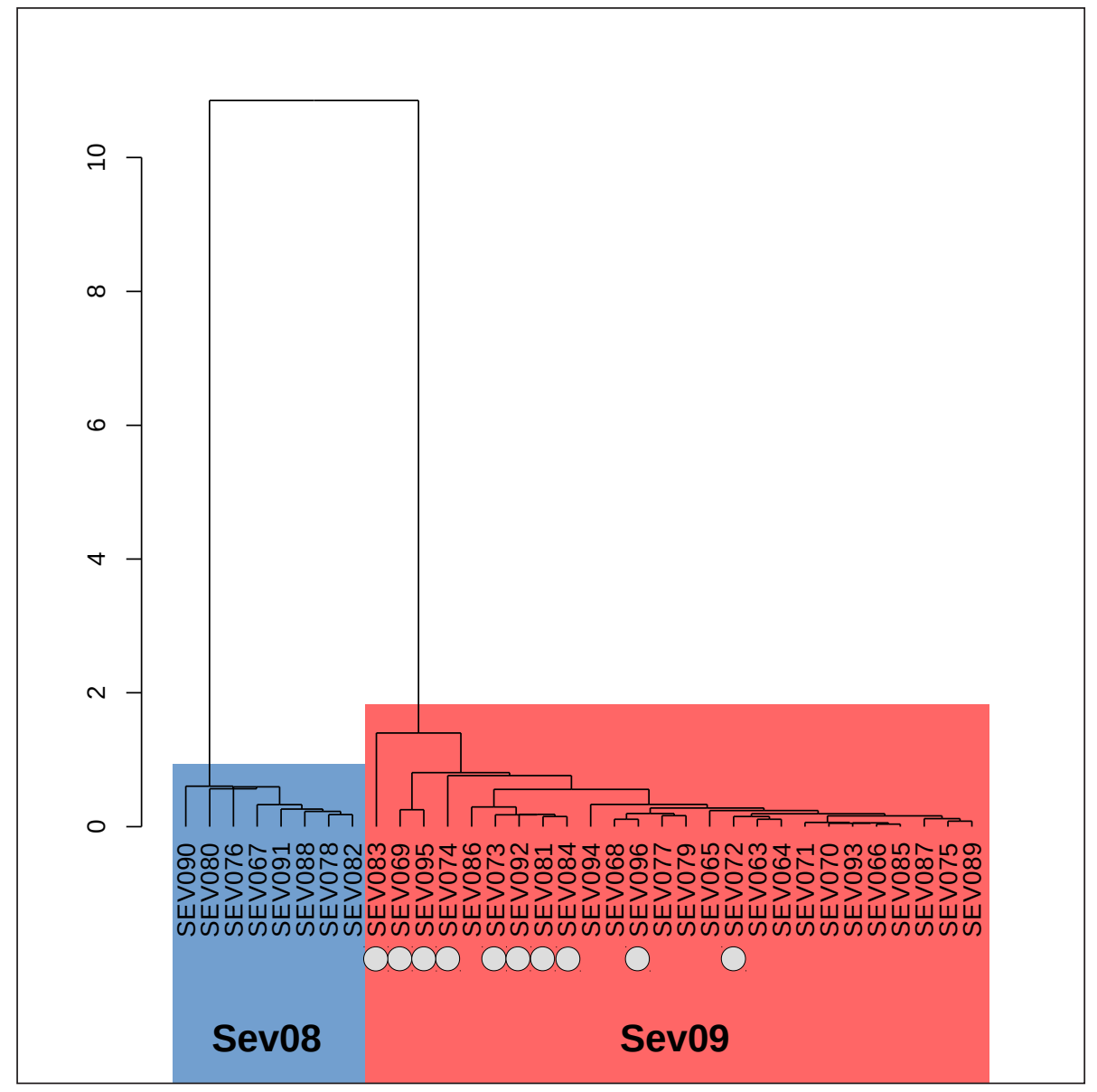

Figura 6. Dendrograma resultante del análisis de clúster sobre las cerámicas de cocina y común vidriada, usando el algoritmo aglomerativo del centroide y la distancia euclidiana al cuadrado sobre sobre la subcomposición $\mathrm{Na}_{2} \mathrm{O}$, $\mathrm{MgO}, \mathrm{Al}_{2} \mathrm{O}_{3}, \mathrm{SiO}_{2}$, $\mathrm{K}_{2} \mathrm{O}, \mathrm{CaO}, \mathrm{TiO}_{2}, \mathrm{~V}, \mathrm{Cr}$, $\mathrm{MnO}, \mathrm{Fe}_{2} \mathrm{O}_{3}, \mathrm{Ni}, \mathrm{Zn}, \mathrm{Sr}$, $\mathrm{Zr}, \mathrm{Nb}, \mathrm{Ba}$ y Ce, con transformación clr. asocian, corresponden a sus equivalentes euclídeos en $R^{d+1}$ (Egozcue y Pawlowsky-Glahn 2011). En el dendrograma los diferentes individuos se unen entre ellos o a grupos ya formados, o se unen grupos a grupos, hasta que todos los individuos quedan unidos entre sí. Cuanto menor es la distancia de la unión respecto a la base del gráfico, mayor es la similitud química entre los individuos o grupos que se unen. El estudio del dendrograma muestra la existencia clara de dos agrupaciones diferentes. De un lado, Sev08 que agrupa todos los individuos de cocina; Por otro lado, Sev09 que agrupa todos los individuos de cerámica común vidriada. Los individuos que aparecen con un círculo (fig. 6) corresponden a aquellos individuos que presentan una alteración que ha afectado, con diferente intensidad, los valores de $\mathrm{Na}_{2} \mathrm{O}$ y $\mathrm{K}_{2} \mathrm{O}$. Esta alteración afecta típicamente a los individuos con sobrecocciones poco severas de cerámicas calcáreas (Buxeda 1999, Buxeda et al. 2002) y será estudiada con detenimiento en la siguiente sección.
Para observar más en detalle la cerámica común vidriada, se realiza un nuevo análisis de conglomerados excluyendo las cerámicas de cocina, usando el algoritmo aglomerativo del centroide y la distancia euclidiana al cuadrado. Además, el análisis se realiza sobre la subcomposición $\mathrm{MgO}, \mathrm{Al}_{2} \mathrm{O}_{3}, \mathrm{SiO}_{2}, \mathrm{CaO}, \mathrm{TiO}_{2}, \mathrm{~V}, \mathrm{Cr}$, $\mathrm{MnO}, \mathrm{Fe}_{2} \mathrm{O}_{3}, \mathrm{Ni}, \mathrm{Zn}, \mathrm{Sr}, \mathrm{Zr}, \mathrm{Nb}, \mathrm{Ba}$ y Ce con transformación clr, excluyendo así el $\mathrm{Na}_{2} \mathrm{O}$ y el $\mathrm{K}_{2} \mathrm{O}$ que presentan alteraciones en un número significativo de cerámicas comunes vidriadas (11 de 26). El estudio del dendrograma resultante (fig. 7) muestra una estructura dentro de esta agrupación Sev09 en tres subgrupos (a, b y c). Esta estructura en subgrupos podría indicar la existencia de tres producciones diferentes en cerámica común vidriada, aunque el número limitado de individuos analizados aconseja, por el momento, tratar toda la cerámica común vidriada como una única agrupación. También se puede observar que hay tres individuos (SEV074, SEV083 y SEV094) que ocupan situaciones particulares, sin quedar bien clasificados en esta estructura en 
Figura 7. Dendrograma resultante del análisis de clúster sobre la cerámica común vidriada, usando el algoritmo aglomerativo del centroide y la distancia euclidiana al cuadrado sobre sobre la subcomposición $\mathrm{MgO}$, $\mathrm{Al}_{2} \mathrm{O}_{3}, \mathrm{SiO}_{2}, \mathrm{CaO}, \mathrm{TiO}_{2}, \mathrm{~V}$, $\mathrm{Cr}, \mathrm{MnO}, \mathrm{Fe}_{2} \mathrm{O}_{3}, \mathrm{Ni}, \mathrm{Zn}$, $\mathrm{Sr}, \mathrm{Zr}, \mathrm{Nb}, \mathrm{Ba}$ y Ce, con transformación clr.

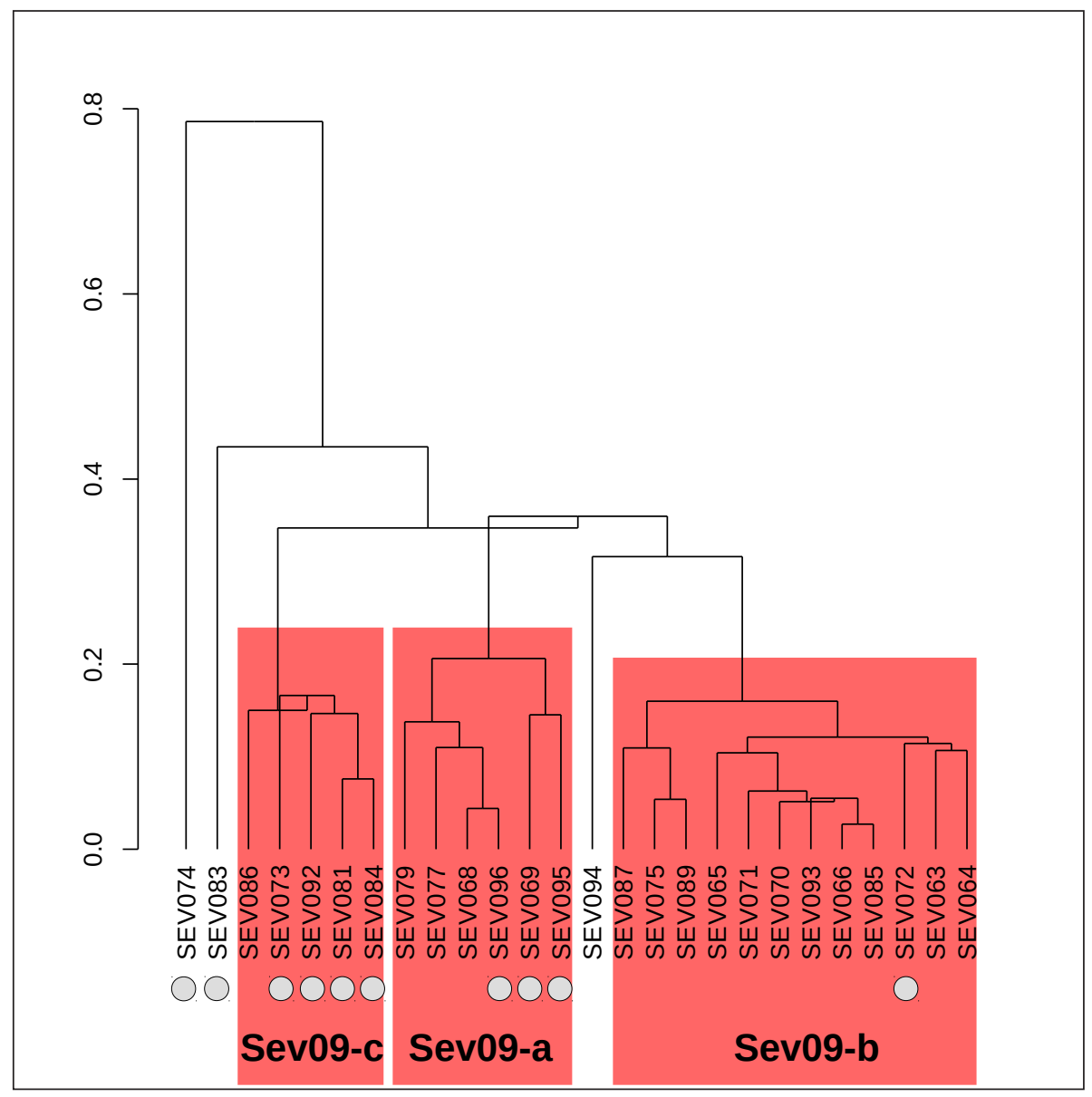

tres subgrupos. Estas muestras se diferencian del resto por algunas diferencias en algunos componentes, aunque las tres, en conjunto, presentan contenidos de $\mathrm{Zn}$ superiores al resto de individuos (Tabla 2). Por lo que respecta a los subgrupos Sev09-a, Sev09-b y Sev09-c, sus diferencias se centran especialmente en los contenidos de $\mathrm{MnO}$ (inferiores en Sev09-a), así como en $\mathrm{CaO}$, $\mathrm{Al}_{2} \mathrm{O}_{3}, \mathrm{y} \mathrm{Zr}$, como ya se observaba en la matriz de diagramas de dispersión (fig. 5, tabla 2).

Arqueológicamente, el grupo Sev08 se corresponde con las cerámicas de cocina, mientras que Sev09 engloba todas las cerámicas comunes vidriadas (Tabla 3). Aunque el reducido número de individuos analizados no permite muchas conclusiones, sí que se puede destacar que los subgrupos de cerámica común vidriada (Sev09) no se corresponden con tipologías específicas. Con todo, el grupo Sev09-c es el único que se compone únicamente por piezas de vajilla para la mesa, sin que tenga ni bacines, ni lebrillos, ni el cangilón. Sin embargo, el hecho de que este subgrupo represente únicamente a 5 individuos obliga, más si cabe, a relativizar estos resultados. Finalmente, los tres individuos cerámicos que no se integran en ningún grupo son un jarrito (SEV074), una jarra (SEV083) y un lebrillo (SEV094).

Finalmente, se han comparado los resultados obtenidos sobre estas cerámicas de cocina y comunes vidriadas con los ya existente en nuestro banco de datos, que abarcan mayoritariamente loza ( 88 individuos, de ellos 8 con decoración de cuerda seca) y contenedores de transporte (43), además de 12 azulejos, 3 morteros y 3 trébedes (Iñañez 2007; Ferrer et al. 2013, 2015a; Buxeda et al. 2015). Todos estos individuos se datan, con variaciones, entre la segunda mitad del s. XV y el s. XVII. El nuevo conjunto presenta, pues, un total de 183 individuos y su matriz de variación composicional indica que la variación total es de 1.02 , siendo el $\mathrm{CaO}$ el componente que introduce una mayor variabilidad $\left(\mathrm{vt} / \tau_{\mathrm{i}}<0.3\right)$, mientras que $\mathrm{Sr}, \mathrm{K}_{2} \mathrm{O}, \mathrm{Na}_{2} \mathrm{O}, \mathrm{Zr}$ y Ce son los otros componentes que introducen una mayor variabilidad $\left(0.3<\mathrm{vt} / \tau_{. \mathrm{i}}<0.5\right)$ (Fig. 8$)$. 
Tabla 2. Media ( $\overline{\mathrm{x}}$ ) y desviación estándar (s) de los resultados químicos según los grupos propuestos, junto con los individuos aislados SEV074, SEV083 y SEV094 (datos normalizados).

\begin{tabular}{|c|c|c|c|c|c|c|c|c|c|c|c|}
\hline & \multicolumn{2}{|c|}{$\begin{array}{l}\text { Sev08 } \\
(\mathrm{n}=8)\end{array}$} & \multicolumn{2}{|c|}{$\begin{array}{l}\text { Sev09-a } \\
(\mathrm{n}=6)\end{array}$} & \multicolumn{2}{|c|}{$\begin{array}{l}\text { Sev09-b } \\
(n=12)\end{array}$} & \multicolumn{2}{|c|}{$\begin{array}{l}\text { Sev09-c } \\
(\mathrm{n}=5)\end{array}$} & \multirow[t]{2}{*}{ SEV074 } & \multirow[t]{2}{*}{ SEV094 } & \multirow[t]{2}{*}{ SEV083 } \\
\hline & $\overline{\mathrm{x}}$ & s & $\overline{\mathrm{x}}$ & s & $\overline{\mathrm{x}}$ & s & $\overline{\mathrm{x}}$ & s & & & \\
\hline $\mathrm{Na}_{2} \mathrm{O}(\%)$ & 0.31 & 0.05 & 0.76 & 0.17 & 0.79 & 0.13 & 0.89 & 0.1 & 0.75 & 0.75 & 1.83 \\
\hline $\mathrm{MgO}(\%)$ & 0.85 & 0.09 & 3.49 & 0.33 & 3 & 0.36 & 2.82 & 0.36 & 2.23 & 2.72 & 3.04 \\
\hline $\mathrm{Al}_{2} \mathrm{O}_{3}(\%)$ & 13.66 & 1.19 & 12.53 & 0.61 & 13.11 & 0.79 & 12.48 & 0.34 & 12.13 & 13.41 & 15.15 \\
\hline $\mathrm{SiO}_{2}(\%)$ & 76.59 & 0.7 & 53.45 & 2.31 & 55.47 & 1.24 & 55.25 & 0.38 & 58.53 & 53.47 & 55.23 \\
\hline $\mathrm{K}_{2} \mathrm{O}(\%)$ & 1.68 & 0.18 & 1.97 & 0.46 & 2.73 & 0.2 & 1.8 & 0.31 & 2.30 & 2.83 & 1.30 \\
\hline $\mathrm{CaO}(\%)$ & 1.23 & 0.28 & 21.93 & 1.92 & 18.75 & 0.91 & 21.01 & 0.86 & 18.33 & 20.54 & 16.62 \\
\hline $\mathrm{TiO}_{2}(\%)$ & 0.59 & 0.04 & 0.7 & 0.02 & 0.73 & 0.03 & 0.7 & 0.01 & 0.70 & 0.73 & 0.77 \\
\hline $\mathrm{V}(\mathrm{ppm})$ & 89 & 5 & 102 & 15 & 97 & 10 & 92 & 6 & 93 & 94 & 118 \\
\hline $\mathrm{Cr}$ (ppm) & 74 & 11 & 81 & 8 & 74 & 9 & 79 & 5 & 17 & 82 & 103 \\
\hline $\mathrm{MnO}(\%)$ & 0.02 & 0.01 & 0.06 & 0.004 & 0.09 & 0.005 & 0.08 & 0.001 & 0.08 & 0.09 & 0.09 \\
\hline $\mathrm{Fe}_{2} \mathrm{O}_{3}(\%)$ & 4.97 & 0.48 & 4.96 & 0.24 & 5.17 & 0.34 & 4.82 & 0.23 & 4.74 & 5.31 & 5.77 \\
\hline $\mathrm{Ni}(\mathrm{ppm})$ & 26 & 3 & 30 & 2 & 34 & 3 & 29 & 3 & 25 & 35 & 41 \\
\hline $\mathrm{Zn}(\mathrm{ppm})$ & 62 & 13 & 86 & 8 & 97 & 11 & 128 & 21 & 189 & 162 & 164 \\
\hline $\mathrm{Sr}(\mathrm{ppm})$ & 75 & 11 & 455 & 40 & 414 & 37 & 532 & 44 & 637 & 420 & 512 \\
\hline $\mathrm{Zr}$ (ppm) & 272 & 47 & 220 & 10 & 213 & 21 & 238 & 5 & 249 & 200 & 199 \\
\hline $\mathrm{Nb}$ (ppm) & 10 & 1 & 12 & 0.86 & 13 & 0.76 & 11 & 1 & 12 & 12 & 13 \\
\hline $\mathrm{Ba}(\mathrm{ppm})$ & 335 & 5 & 363 & 2 & 484 & 3 & 477 & 5 & 627 & 463 & 586 \\
\hline $\mathrm{Ce}(\mathrm{ppm})$ & 68 & 8 & 96 & 18 & 77 & 5 & 120 & 15 & 89 & 73 & 125 \\
\hline
\end{tabular}

Tabla 3. Correspondencias entre tipología y grupos definidos en el presente estudio.

\begin{tabular}{|c|c|c|c|c|c|c|c|c|c|c|c|}
\hline GRUPO & bacín & lebrillo & jarra & jarro & jarrito & jarrita & cangilón & olla & cazuela & informe & TOTAL \\
\hline Sev08 & & & & & & & & 1 & 6 & 1 & 8 \\
\hline Sev09-a & & 3 & 1 & & 2 & & & & & & 6 \\
\hline Sev09-b & 3 & 5 & 1 & 1 & 1 & & 1 & & & & 12 \\
\hline Sev09-c & & & & 2 & 1 & 2 & & & & & 5 \\
\hline SEV074 & & & & & 1 & & & & & & 1 \\
\hline SEV083 & & & 1 & & & & & & & & 1 \\
\hline SEV094 & & 1 & & & & & & & & & 1 \\
\hline TOTAL & 3 & 9 & 3 & 3 & 5 & 2 & 1 & 1 & 6 & 1 & 34 \\
\hline
\end{tabular}




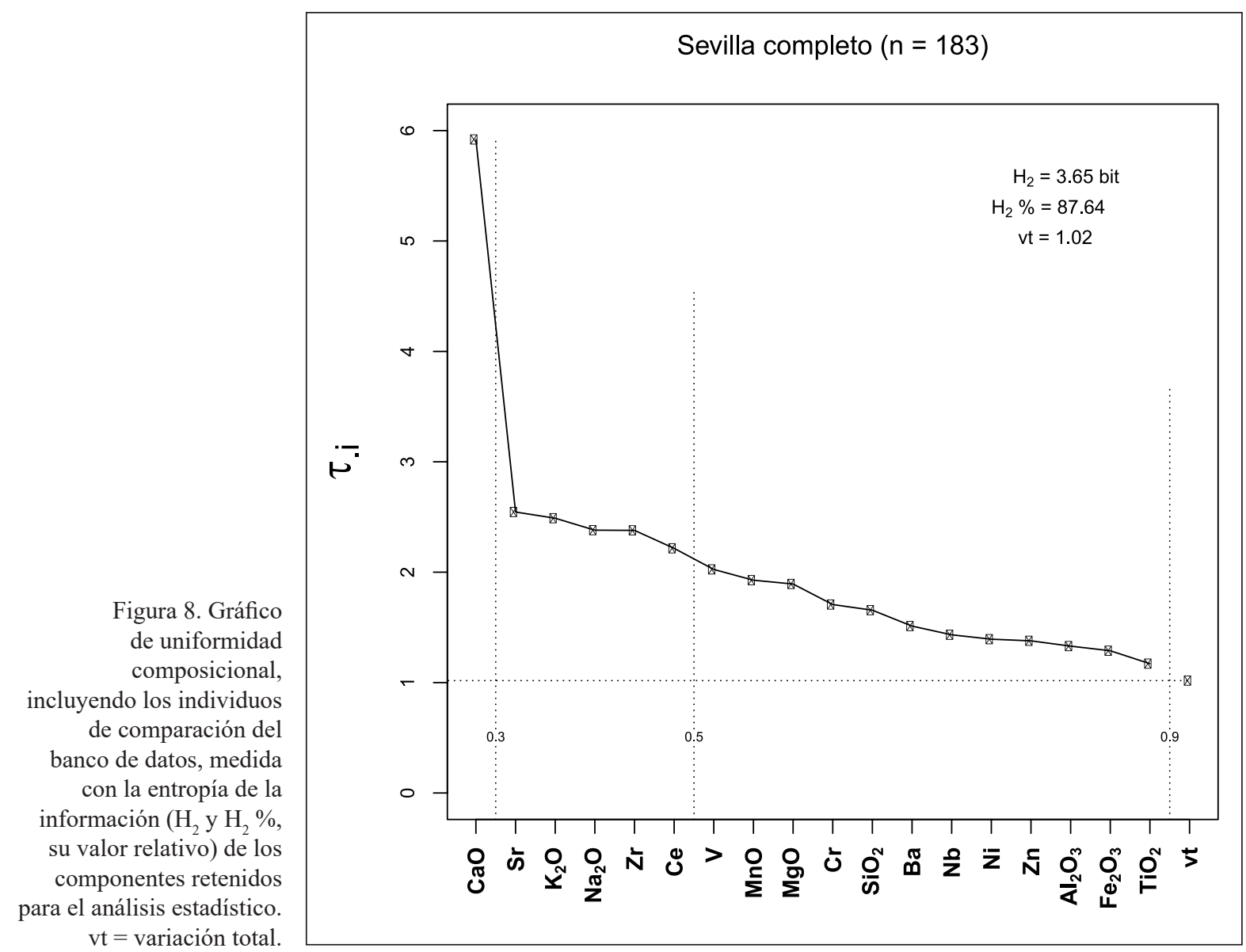

Como resumen de esta comparación, ofrecemos el dendrograma resultante del análisis de conglomerados realizado con la distancia euclidiana al cuadrado y el algoritmo aglomerativo del centroide sobre la subcomposición $\mathrm{Na}_{2} \mathrm{O}, \mathrm{MgO}, \mathrm{Al}_{2} \mathrm{O}_{3}, \mathrm{SiO}_{2}, \mathrm{~K} 2 \mathrm{O}, \mathrm{CaO}, \mathrm{TiO}_{2}, \mathrm{~V}$, $\mathrm{Cr}, \mathrm{MnO}, \mathrm{Fe}_{2} \mathrm{O}_{3}, \mathrm{Ni}, \mathrm{Zn}, \mathrm{Sr}, \mathrm{Zr}, \mathrm{Nb}, \mathrm{Ba}$ y $\mathrm{Ce}$, con transformación clr (fig. 9). Hay que destacar, sin embargo, que este análisis de aglomerados no se ha realizado sobre los 183 individuos, sino sobre los 97 individuos que no presentan alteraciones, ni fases minerales de difícil interpretación en su composición debidas a posibles procesos de meteorización postdeposicional. En el conjunto aquí estudiado, esto afecta a los 10 individuos de cerámica común vidriada (SEV069, 72, 73, $74,81,83,84,92,95$ y 96) indicados con un círculo en las fig. 6 y 7, que presentan analcima, además del individuo SEV086, que presenta aragonito. El estudio del dendrograma muestra claramente la gran diferencia existente entre las producciones de cocina, correspondientes a cerámicas poco calcáreas $(\mathrm{CaO}<5 \%-6 \%)$, frente a todas las otras producciones caracterizadas hasta el presente, correspondientes todas ellas a cerámicas calcáreas ( $5 \%-6 \%<\mathrm{CaO}<15 \%$ - $25 \%$ ). Los resultados así obtenidos permiten comprobar cómo, en este período, las producciones de Sevilla presentan una variedad de materias primas y recetas de preparación de las pastas cerámicas según la funcionalidad de las piezas. Este resultado era ya esperado para las cerámicas de cocina, cuyos requerimientos técnicos condicionan en gran medida los productos a manufacturar (Hein et al. 2008), pero se ha comprobado igualmente para las cerámica comunes vidriadas que no emplean los materiales utilizados por la loza ni por los contenedores de transporte.

\subsection{Caracterización mineralógica}

El estudio sobre la caracterización mineralógica permite la definición de fábricas dentro de cada grupo químico definido, entendiendo como fábrica la distribución, frecuencia, forma, tamaño y composición 


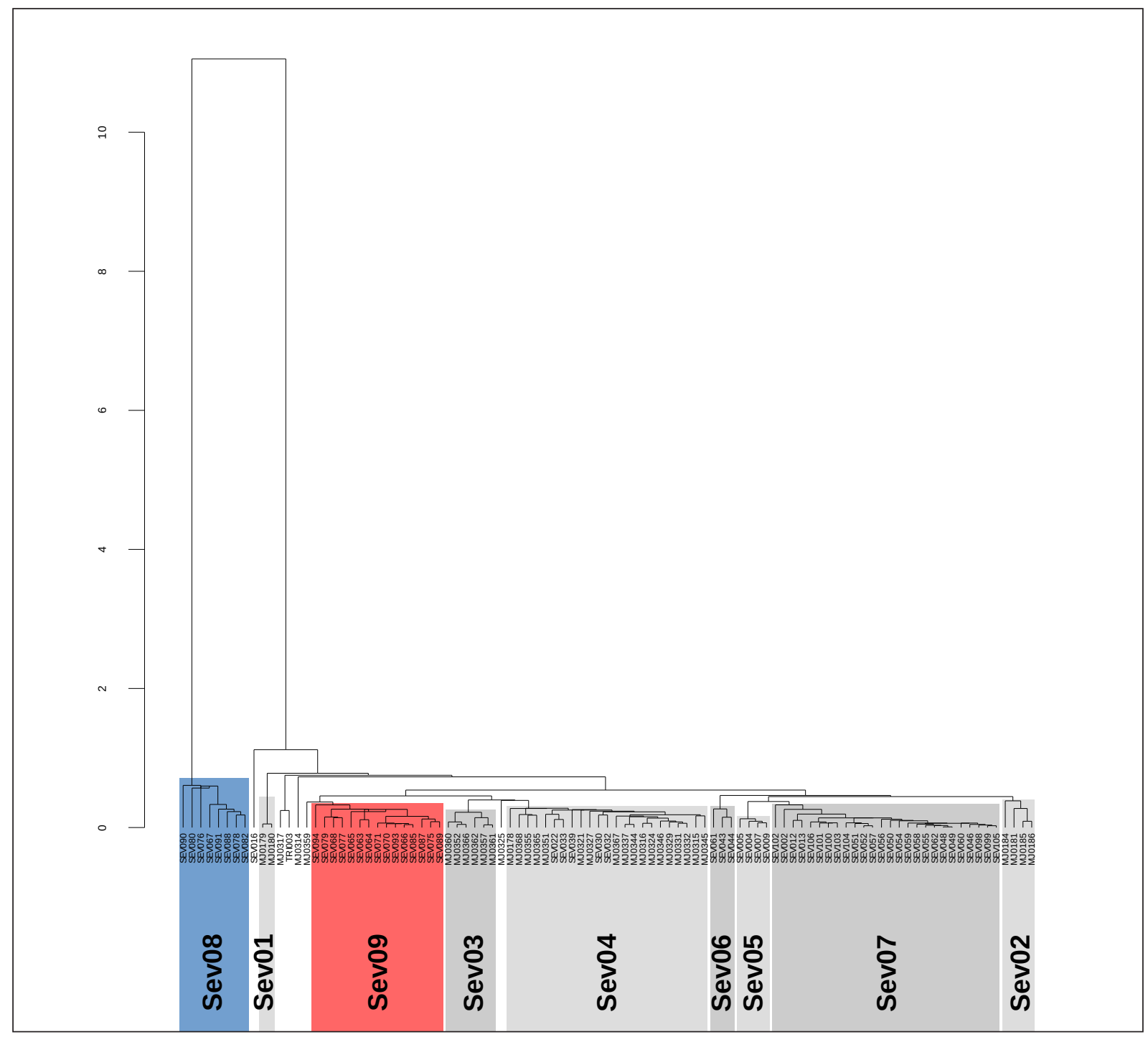

Figura 9. Dendrograma resultante del análisis de clúster, incluyendo los individuos de comparación del banco de datos, usando el algoritmo aglomerativo del centroide y la distancia euclidiana al cuadrado sobre sobre la subcomposición $\mathrm{Na}_{2} \mathrm{O}, \mathrm{MgO}_{2} \mathrm{Al}_{2} \mathrm{O}_{3}$, $\mathrm{SiO}_{2}, \mathrm{~K}_{2} \mathrm{O}, \mathrm{CaO}, \mathrm{TiO}_{2}, \mathrm{~V}, \mathrm{Cr}, \mathrm{MnO}, \mathrm{Fe}_{2} \mathrm{O}_{3}, \mathrm{Ni}, \mathrm{Zn}, \mathrm{Sr}, \mathrm{Zr}, \mathrm{Nb}, \mathrm{Ba}$ y Ce, con transformación clr.

de los componentes de una cerámica (Buxeda et al. 1995: 49). Estas fábricas, a su vez, han de permitir establecer, a partir de las fases primarias y de cocción (Maggetti 1981, 1982), las escalas mineralógicas sobre las que inferir las temperaturas de cocción equivalentes. El término 'temperatura de cocción equivalente' (TCE) se usa aquí en vez del término 'temperatura de cocción', que aparece frecuentemente en la literatura arqueométrica, ya que no es posible, en realidad, estimar la temperatura de cocción empleada en la fabricación original de las cerámicas ya que no es posible controlar todos los factores que afectan a una cocción cerámica y que, en su mayor parte, permanecen desconocidos. Este hecho fue inicialmente observado por Roberts (1963) quien acuñó el término de temperatura de cocción equivalente como aquella temperatura estimada a partir de unos experimentos determinados bajo control y cuyo resultado es similar al que se observa en la cerámica en estudio.

En primer lugar hemos realizado el diagrama ternario del sistema $\mathrm{CaO}-\mathrm{Al}_{2} \mathrm{O}_{3}-\mathrm{SiO}_{2}$ y el triángulo cerámico (fig. 10) para observar en qué triángulos de equilibrio termodinámico quedan situados los individuos en estudio. Estos triángulos determinan las fases minerales 


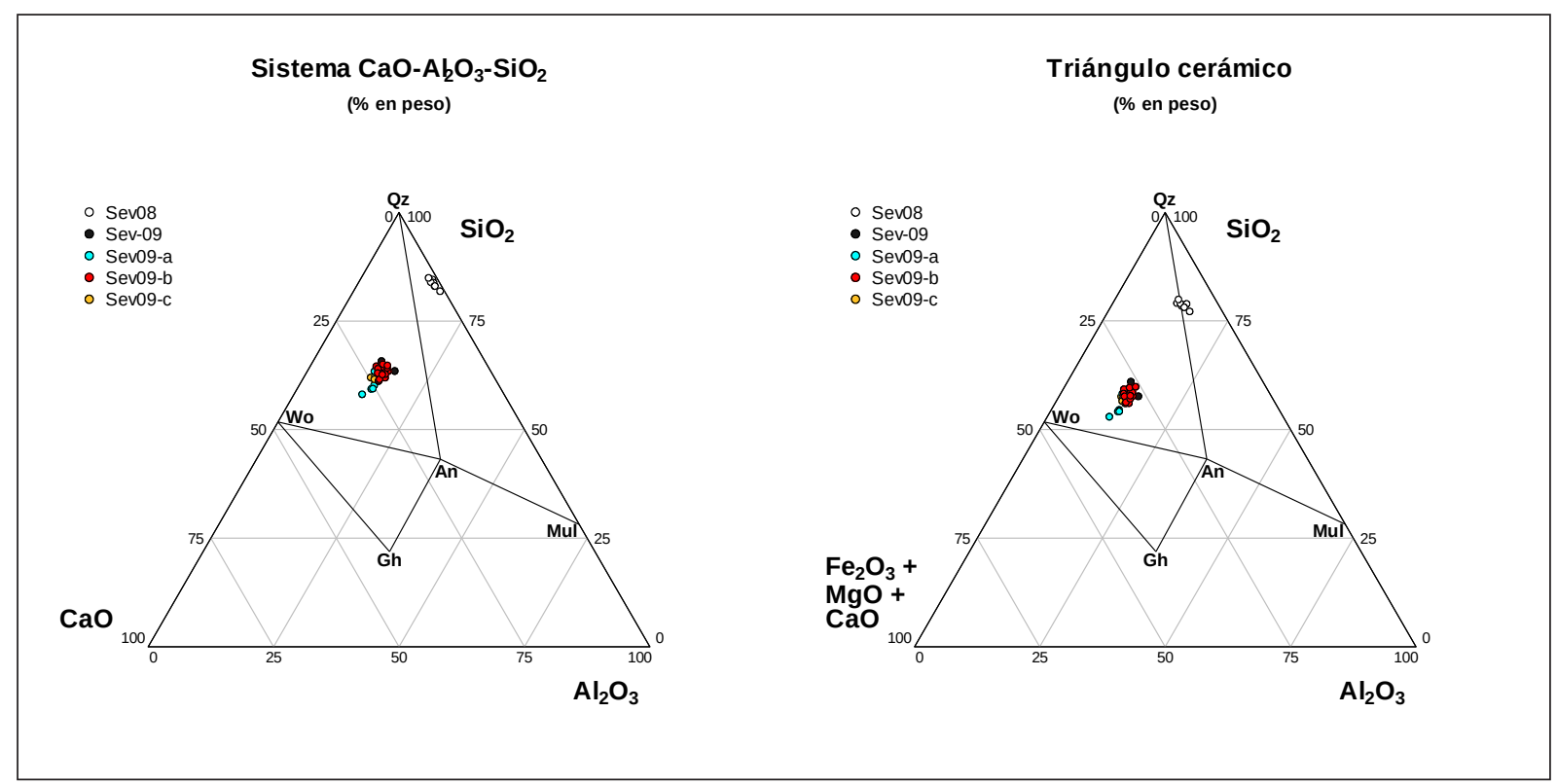

Figura 10. Izquierda: Diagrama ternario del sistema CaO- $\mathrm{Al}_{2} \mathrm{O}_{3}-\mathrm{SiO}_{2}$. Derecha: Triangulo ceràmico del sistema $\left(\mathrm{CaO}+\mathrm{Fe}_{2} \mathrm{O}_{3}+\right.$ $\mathrm{MgO})-\mathrm{Al}_{2} \mathrm{O}_{3}-\mathrm{SiO}_{2}$. An: anortita $\left.\left(\mathrm{Ca}_{2} \mathrm{Al}_{2} \mathrm{Si}_{2} \mathrm{O}_{8}\right]\right)$; Gh: gehlenita $\left(\mathrm{Ca}_{2} \mathrm{Al}(\mathrm{Si}, \mathrm{Al})_{2} \mathrm{O}_{7}\right)$; Mul: mullita $\left(\mathrm{Al}_{6}\left[\mathrm{Si}_{2} \mathrm{O}_{13}\right]\right) ;$ Qz: cuarzo $\left(\mathrm{SiO}_{2}\right)$; Wo: wollastonita $\left(\mathrm{CaSiO}_{3}\right)$ (abreviaturas según Whitney y Evans 2010$)$.

que deberían presentarse a medida que la temperatura aumenta, es decir las fases de cocción esperadas.

Tal como observamos en los dos triángulos, hay una separación clara entre las cerámicas calcáreas y las poco calcáreas. La división entre cerámicas calcáreas y poco calcáreas es fundamental por lo que respecta a la técnica de producción cerámica. Normalmente, los altos contenidos de $\mathrm{CaO}$ de una cerámica se deben a una presencia importante de carbonato cálcico, la descomposición del cual se produce a altas temperaturas según:

$$
\mathrm{CaCO}_{3(\mathrm{~s})} \rightarrow \mathrm{CaO}_{(\mathrm{s})}+\mathrm{CO}_{2(\mathrm{~g})}
$$

Esta descomposición favorece la cristalización de calcosilicatos y calcoaluminio-silicatos de alta temperatura, además de la formación de una microestructura celular característica. Aparte de esto, en cocciones con postcocciones oxidantes, las pastas calcáreas pueden favorecen el desarrollo de colores claros a altas temperaturas, debido a la descomposición de la calcita y al desarrollo de piroxenos que pueden incorporar hierro en sus estructuras cristalinas, inhibiendo el desarrollo de la hematites (Maniatis et al. 1981). En cambio, en las pastas poco calcáreas pasa lo contrario, puesto que el gran desarrollo de la hematites produce colores intensos de la pasta de tonos rojizos o anaranjados. Los minerales formados a altas temperaturas, además, son menos y la microestructura adquiere un aspecto denso característico.

En la tabla 4 mostramos las fábricas que hemos diferenciado en cada grupo químico, así como las fases cristalinas, la temperatura de cocción equivalente (TCE) de cada fábrica y las muestras que se incluyen en cada fábrica. Cabe destacar que en el presente estudio no se han realizado experimentos de recocciones, ni termodifractométricos para estimar la TCE de los diferentes grupos definidos. Estas estimaciones se han realizado a partir de datos publicados en otros estudios sobre cerámicas hechas con arcillas de base illítica poco calcáreas y calcáreas, que permiten definir unas evoluciones generales de las escalas mineralógicas (especialmente Maggetti 1981, 1982, Buxeda 1999).

Como se puede observar, para el grupo Sev08 hemos detectado tres fábricas que se diferencian básicamente por la mayor o menor presencia de illita-moscovita. Así, a medida que va en aumento la TCE, la illita-moscovita tiene menos presencia y, finalmente, cristaliza la espinela. De este modo, vemos como las TCE del grupo Sev08 varían de $(850-950){ }^{\circ} \mathrm{C}$ en la Fábrica 1 (fig. 11 inferior), con la presencia del pico de $10 \AA \AA$ de la illita-moscovita, a los $(900-950)^{\circ} \mathrm{C}$ en la Fábrica 2 (fig. 11 centro), en donde no se observa el pico de $10 \AA$ de la illita-moscovita, hasta por encima de $(950-1000)^{\circ} \mathrm{C}$ de la Fábrica 3 (fig. 11 superior), con la cristalización de la espinela. Por 
Tabla 4. Fábricas correspondientes a los grupos químicos Sev08, Sev09-a, Sev09-b y Sev09-c, e individuos aislados, con las fases minerales predominantes, las temperaturas de cocción equivalentes estimadas (TCE) y los individuos que se incluyen en cada fábrica. Afs: feldespato alcalino, Anl: analcima, Cal: calcita, Di: diópsido, Gh: gehlenita, Hem: hematites, Ill: illita-moscovita, Ill*: illita-moscovita sin el pico de $10 \AA$, Pl: plagioclasas, Qz: cuarzo, Spl: espinela (abreviaturas según Whitney y Evans 2010).

\begin{tabular}{|c|c|c|c|r|c|}
\hline Grupo & Fábricas & Fases cristalinas & Muestra & Tce & Total \\
\hline \multirow{4}{*}{ Sev08 } & Fábrica 1 & Ilt, Qz, Afs, Hem & SEV076, 78, 90 & $(850-950)^{\circ} \mathrm{C}$ & 3 \\
\cline { 2 - 7 } & Fábrica 2 & Ilt*, Qz, Afs, Hem & SEV067, 82, 88, 91 & $(900-950)^{\circ} \mathrm{C}$ & 4 \\
\cline { 2 - 7 } & Fábrica 3 & Qz, Afs, Spl, Hem & SEV080 & $>(950-1000)^{\circ} \mathrm{C}$ & 1 \\
\hline \multirow{4}{*}{ Sev09-a } & Fábrica 1 & Ilt, Qz, Afs, Pl, Cal, Gh, Di, Hem & SEV077, 79 & $(850-950)^{\circ} \mathrm{C}$ & 2 \\
\cline { 2 - 7 } & Fábrica 2 & Qz, Afs, Pl, Cal, Gh, Di, Hem & SEV068, 96 & $(1000-1050)^{\circ} \mathrm{C}$ & 2 \\
\cline { 2 - 7 } & Fábrica 3 & Anl, Qz, Afs, Pl, Cal, Gh, Di, Hem & SEV069, 95 & $(1000-1050)^{\circ} \mathrm{C}$ & 2 \\
\hline \multirow{3}{*}{ Sev09-b } & Fábrica 1 & Ilt, Qz, Afs, Pl, Cal, Gh, Di, Hem & SEV066, 75, 85, 89, 93 & $(850-950)^{\circ} \mathrm{C}$ & 5 \\
\cline { 2 - 7 } & Fábrica 2 & Qz, Afs, Pl, Cal, Gh, Di, Hem & SEV063, 64, 65, 70,71, 87 & $(1000-1050)^{\circ} \mathrm{C}$ & 6 \\
\cline { 2 - 7 } & Fábrica 3 & Anl, Qz, Afs, Pl, Cal, Gh, Di, Hem & SEV072 & $(1000-1050)^{\circ} \mathrm{C}$ & 1 \\
\hline \multirow{2}{*}{ Sev09-c } & Fábrica 1 & Arg, Qz, Afs, Pl, Cal, Gh, Di, Hem & SEV086 & $(1000-1050)^{\circ} \mathrm{C}$ & 1 \\
\cline { 2 - 7 } & Fábrica 2 & Anl, Qz, Afs, Pl, Cal, Gh, Di, Hem & SEV073, 81, 84, 92 & $(1000-1050)^{\circ} \mathrm{C}$ & 4 \\
\hline SEV094 & & Ilt, Qz, Afs, Pl, Cal, Gh, Di, Hem & & $(850-950)^{\circ} \mathrm{C}$ & 1 \\
\hline SEV074 & & Anl, Qz, Afs, Pl, Cal, Gh, Di, Hem & & $(1000-1050)^{\circ} \mathrm{C}$ & 1 \\
\hline SEV083 & & Anl, Qz, Afs, Pl, Cal, Gh, Di, Hem & & $(1000-1050)^{\circ} \mathrm{C}$ & 1 \\
\hline Total & & & & & 34 \\
\hline
\end{tabular}

otro lado, para las fábricas que hemos detectado en el grupo de las calcáreas, se diferencian de las poco calcáreas por la presencia de calcita, que puede ser tanto primaria residual como secundaria (Buxeda 1999), y la cristalización de minerales de piroxeno y la gehlenita. En las fábricas de las cerámicas calcáreas también podemos ver cómo a medida que aumenta la temperatura, las fases cristalinas de illita-moscovita van desapareciendo dejando paso a fases cristalinas características de alta temperatura. Estos cambios se ilustran con la evolución observada en el grupo Sev09-b, cuya Fábrica 1 (fig. 12 inferior) presenta ya como fases de cocción el piroxeno y la gehlenita, junto con la presencia del pico de $10 \AA$ de la illita-moscovita, que ya no se observa en la Fábrica 2 (fig. 12 centro). Además, algunas de las fábricas de los grupos calcáreos, como la Fábrica 3 del grupo Sev09-c (fig. 12 superior), se caracterizan por la cristalización de analcima, tal como hemos comentado anteriormente al tratar los datos químicos. La cristalización de analcima es típica de cerámicas calcáreas con sobrecocciones poco severas y es consecuencia del proceso de alteración de los individuos cerámicos durante su etapa postdeposicional. Este proceso comporta una pérdida de $\mathrm{K}_{2} \mathrm{O}$ acompañada por una cristalización de esta zeolita sódica, que supone enriquecimiento de $\mathrm{Na}_{2} \mathrm{O}$ en las muestras al fijar este elemento procedente de las aguas de circulación (Buxeda et al. 2002, Schwedt et al. 2006).

\section{CONCLUSIONES}

Durante el siglo XVI, Sevilla se convirtió en un modelo como ciudad gracias al impulso económico que recibió a partir del comercio con América, pero también gracias a la transformación que la ciudad había tenido en época islámica. Tal como hemos visto, unos de los barrios que se vio más beneficiado por este crecimiento económico fue Triana, el barrio donde se trasladó la gran mayoría de los alfares que ya había en la ciudad y donde se crearon nuevos. La gran ventaja de Triana, y que supieron aprovechar bien los ceramistas, es que la arcilla utilizada para la fabricación de la cerámica se podía conseguir muy cerca, justo en la orilla del río Guadalquivir, reduciendo al mínimo el transporte de la materia prima. Pero los alfareros no solamente se tenían que desplazar poco, sino que la arcilla era de buena calidad. Gracias a todos estos factores, Sevilla se convirtió en un importante centro productor y difusor 
Figura 11. Difractogramas representativos de las fábricas definidas para el Grupo Sev08.

Inferior: Difractograma del individuo SEV076 representativo de la Fábrica 1. Centro: Difractograma del individuo SEV088 representativo de la Fábrica 2. Superior: Difractograma del individuo SEV080 representativo de la Fábrica 3. La escala en ${ }^{\circ} 2 \theta$. Afs: feldespato alcalino; Hem: hematita; Ill: illita-moscovita; Qz: cuarzo;

Spl: espinela (abreviaturas según Whitney y Evans 2010).

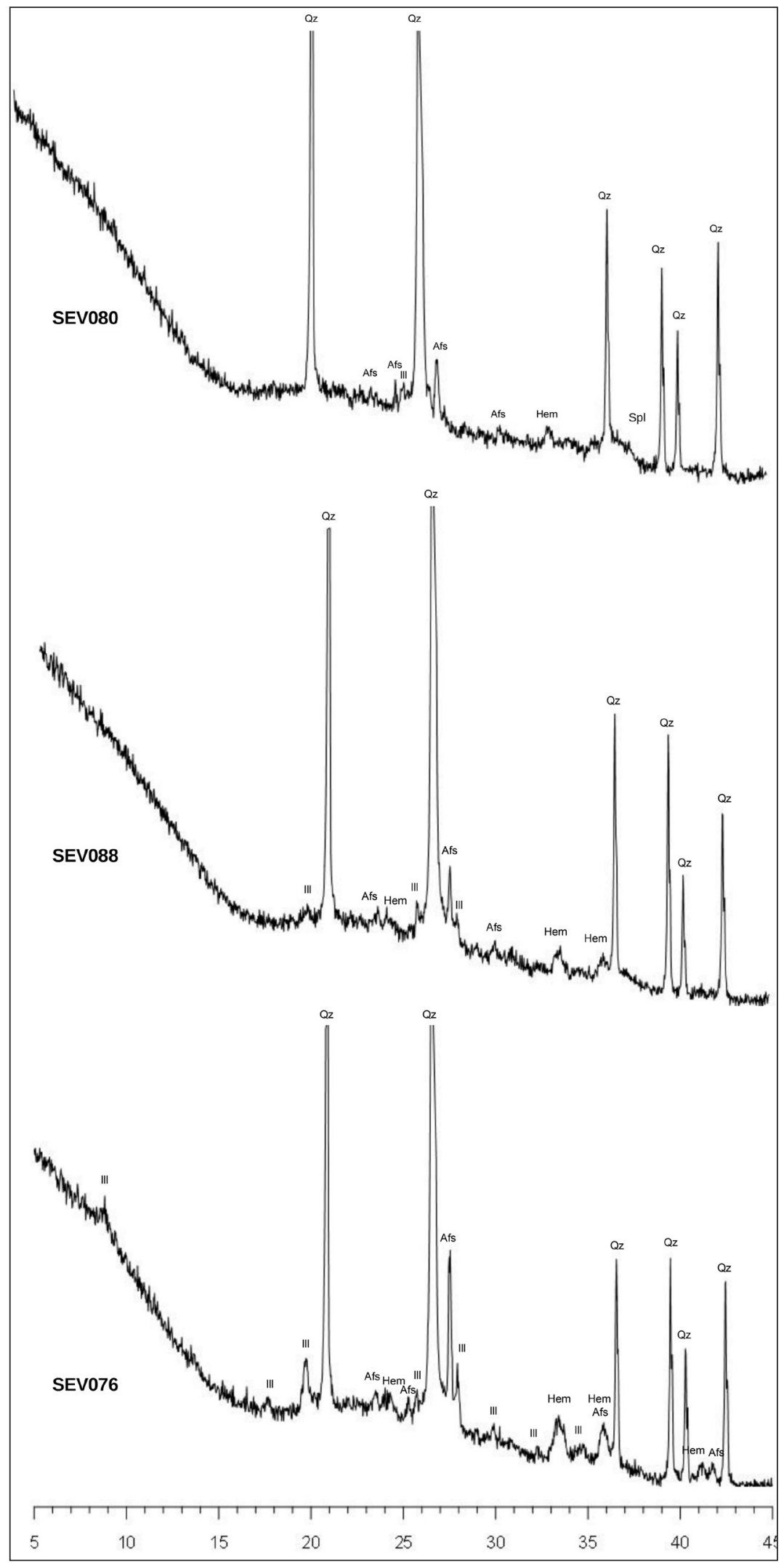




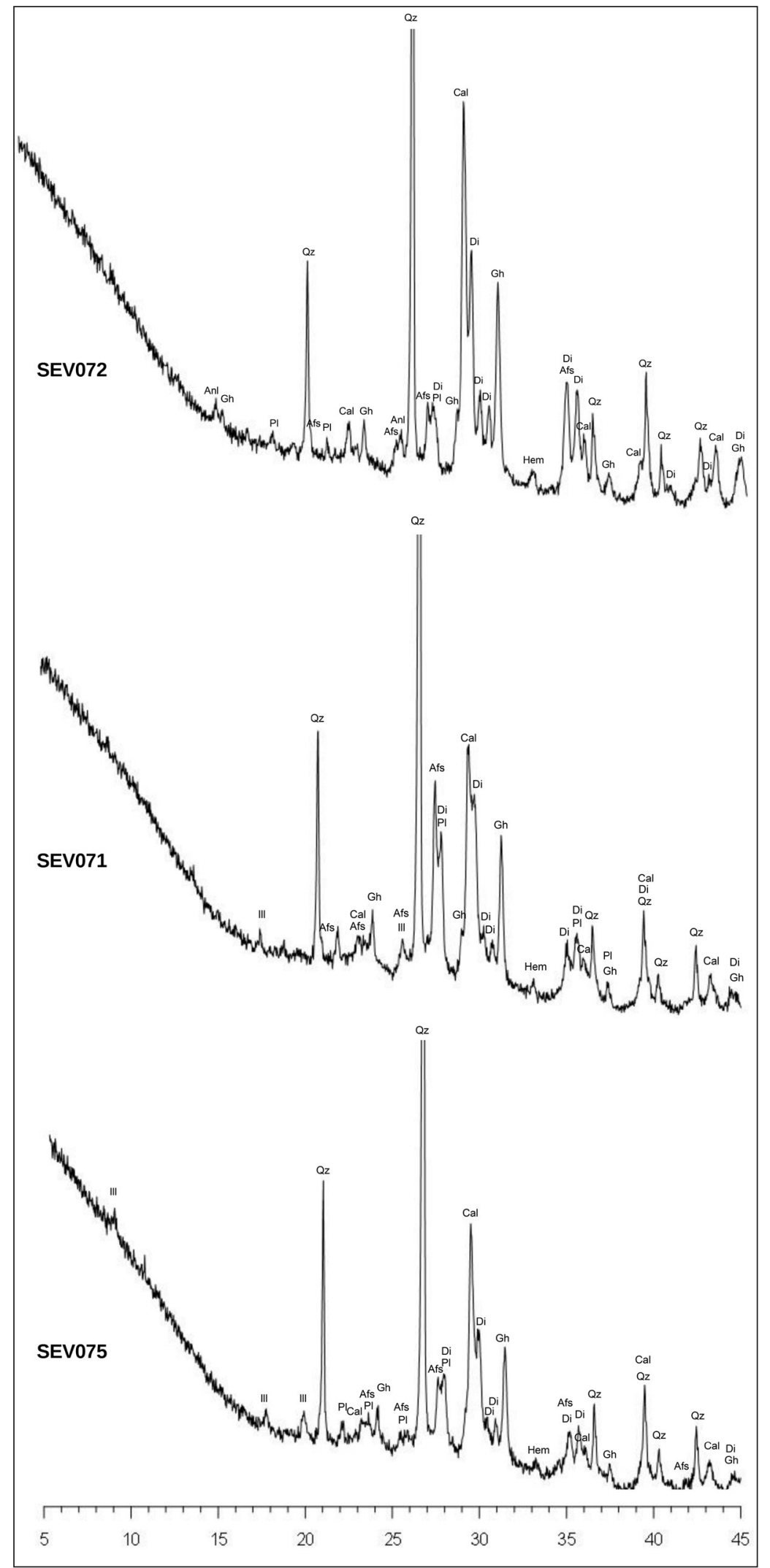

SPAL 26 (2017): 259-280
Figura 12. Difractogramas

representativos de las fábricas definidas para los grupos calcáreos. Inferior: Difractograma del individuo SEV075 representativo de la Fábrica 1 del grupo Sev09-b. Centro: Difractograma del individuo SEV071 representativo de la Fábrica 2 del grupo Sev09-b. Superior: Difractograma del individuo SEV072 representativo de la Fábrica 3 del grupo Sev09-b. La escala en ${ }^{\circ} 2 \theta$. Afs: feldespato alcalino; Anl: analcima; $\mathrm{Cal}$ : calcita; Di: diópsido (piroxeno); Gh: gehlenita; Hem: hematita; Ill: illitamoscovita; Pl: plagioclasa; Qz: cuarzo (abreviaturas según Whitney y Evans 2010)

ISSN: 1133-4525 ISSN-e: 2255-3924 http://dx.doi.org/10.12795/spal.2017i26.11 
de todo tipo de cerámica, desde mayólica hasta cerámica de uso doméstico y de transporte.

El objetivo principal de este trabajo era complementar la visión de la gran complejidad de la producción cerámica que los estudios más recientes han empezado a evidenciar. Para ello, realizamos la caracterización arqueométrica de 34 muestras de loza basta procedentes de la ciudad de Sevilla, en concreto cerámica común vidriada y cerámica de cocina vidriada. Hasta el momento, se habían hecho pocos análisis de cerámica vidriada común sevillana, mientras la cerámica de cocina nunca antes se había caracterizado arqueométricamente.

Gracias a los análisis, hemos podido ver cómo estos tipos de cerámica se diferenciaban entre ellos. De este modo, químicamente se han distinguido dos grandes grupos, siendo la cerámica de cocina vidriada (Sev08) poco calcárea y la cerámica común vidriada calcárea. Mientras las cerámicas de cocina parecen formar una sola producción, las cerámicas comunes parecen dividirse en tres subgrupos diferentes (Sev09-a, Sev09-b y Sev09-c). Entre ellos, el subgrupo Sev09-c está formado exclusivamente por ejemplares de vajilla de mesa (jarros, jarras, jarritos y jarritas), hecho que nos hace pensar que la arcilla escogida para fabricar estas piezas dependía de la funcionalidad de la cerámica. Aún así, en los otros dos subgrupos también encontramos cerámica de mesa, junto con lebrillos en el subgrupo Sev09-a y lebrillos, bacines y un cangilón en el subgrupo Sev09-b. A partir de aquí, deducimos que las piezas de cerámica común que hemos analizado podrían corresponder a tres producciones distintas. Aun así, a causa del número limitado de muestras analizadas, tendremos que corroborar esta hipótesis en futuros trabajos.

Técnicamente, y respecto a las cerámicas de cocina, la utilización de arcillas poco calcáreas con numerosas inclusiones no plásticas se debe a su mejor adaptación para ser usadas en la cocina en contacto con el fuego, ya que estas características optimizan la conductividad térmica y evitan la propagación de fracturas causadas por el choque térmico (Hein et al. 2009). Aparte de esto, a pesar de las diferencias observadas en los resultados químicos, los ceramistas habrían utilizado una misma técnica para la fabricación de todas las cerámicas, es decir, que se podrían cocer en el mismo horno, con un mismo proceso de cocción y postcocción oxidante/reductor-oxidante (Picon 1973: 65) y con tendencia a utilizar una temperatura alrededor de los $950^{\circ} \mathrm{C}$. Aún así, podemos encontrar alguna excepción con temperaturas por debajo de ésta. Además, podemos deducir que el ceramista procuraba que la pasta de la cerámica para la mesa fuese más fina y prácticamente de color claro, ya que de este modo era más fácil producir el vidriado en el tono deseado. En cambio, con todas aquellas cerámicas de uso doméstico que no tenían presencia en la mesa, no importaba si el color de la matriz no era tan blanco, porque a pesar de que también llevaban vidriado, no se buscaba que su aspecto fuese minucioso, sino que se priorizaba su funcionalidad.

Finalmente, el contraste de nuestros resultados con la base de datos del proyecto Tecnolonial nos ha permitido observar que, aún ampliando el muestreo, las cerámicas de cocina continúan siendo una producción completamente diferente al resto. Pero también hemos visto como las mayólicas, las cerámicas de transporte y las cerámicas de uso doméstico corresponden a producciones diferentes, por tanto, su funcionalidad es un factor clave para la elección de la materia prima.

\section{Agradecimientos}

Los análisis por fluorescencia de rayos $\mathrm{X}$ y difracción de rayos $\mathrm{X}$ se han realizado en los Centres Científics i Tecnològics de la Universitat de Barcelona (CCiTUB). Este trabajo se incluye en el proyecto "Impacto tecnológico en el nuevo mundo colonial. Cambio cultural en arqueología y arqueometría cerámica" (Tecnolonial) (HAR2012-33784) financiado por el Ministerio de Economía y Competitividad. Cristina Fernández de Marcos García tiene una beca del programa Ajuts de Personal Investigador predoctoral en Formació (APIF) de la Universitat de Barcelona.

\section{BIBLIOGRAFÍA}

Aitchison, J. (1986): The statistical analysis of compositional data, Monographs on Statistics and Applied Probability. London-New York, Chapman and Hall.

Aitchison, J. (2005): A concise guide to compositional data analysis. 2nd Compositional Data Analysis Workshop - CoDaWork'05. Girona, Universitat de Girona.

Amores Carredano, F. (1998): "La arqueología en la recuperación de la Cartuja de Sevilla. 1986-1992". PH: Boletín del Instituto Andaluz del Patrimonio Histórico 24: 68-81.

Amores Carredano, F. (1999): "La Arqueología en la Recuperación de la Cartuja de Sevilla”, en R. Mar, J. Ruiz de Arbulo y E. Subías (ed.), Recuperar la Memòria Urbana. L'Arqueologia en la Rehabilitació de Les Ciutats Històriques: seminari: 
Tarragona, 27 i 28 de febrer de 1997: 47-74. Tarragona, Universitat Rovira i Virgili.

Amores Carredano, F. y Chisvert Jiménez, N. (1993): "Tipología de la cerámica bajomedieval y moderna sevillana (s. XV-XVIII): I, la loza quebrada de relleno de bóvedas". SPAL: Revista de prehistoria y arqueología de la Universidad de Sevilla 2: 269-328.

Amores Carredano, F.; Chisvert Jiménez, N.; Fernández Ruiz, R.; Fuentes Bonavita, A. M.; Hunt Ortiz, M.; Izarzugaza Lizárraga, I.; Jiménez Barrientos, J. C.; López Torres, J.; Mantero Tocino, A.; Pozo Blázquez, F.; Rodríguez Achútegui, C.; Rodrigues de Oliveira, S.; Rueda Galán, M.; Salido Campos, D.; Vargas Durán, M. A. y Vera Reina, M. (1997): "Informe sobre las actuaciones arqueológicas de apoyo a la restauración en La Cartuja de Sevilla (1987-1992). Anuario Arqueológico de Andalucía 1993, vol. III: 594-608.

Amores Carredano, F. y López Torres, P. (2009): “Las cerámicas finas -alcazarras blancas- de Sevilla en la Edad Moderna: la expresión barroca de una tradición almohade", en R. Cruz-Auñón Briones y E. Ferrrer Albelda (coord.), Estudios de Prehistoria y Arqueología en homenaje a Pilar Acosta Martínez: 563-573. Sevilla, Universidad de Sevilla.

Barceló-Vidal, C., Martín-Fernández, J. y PawlowskyGlahn, V. (2001): "Mathematical foundations of compositional data analysis", en G. Ross (ed.), Proceedings of IAMG'01 - The annual meeting of the International Association for Mathematical Geology, 6-12 September 2001, Cancun, Mèxic: 1-20. Cancún.

Blackman, M. J., Fournier, P. y Bishop, R. L. (2006): "Complejidad e interacción social en el México colonial: identidad, producción, intercambio y consumo de lozas de tradición ibérica, con base en análisis de activación neutrónica". Cuicuilco 13(36): 203-222.

Buxeda i Garrigós, J. (1999): “Alteration and Contamination of Archaeological Ceramics: The Perturbation Problem". Journal of Archaeological Sciences 26: 295-313.

Buxeda i Garrigós, J. (2001): “L'estadística i la seva aplicació en els estudis de provinença dels materials arqueològics”, en F. Gracia, J. M. Gurt, C. Carreras y G. Munilla (eds.), Jornades d'Arqueologia i Tecnologies de la Informació i la Comunicació: Recerca, Docència i Difusió, Arqueo-Mediterrània 7: 71-92. Barcelona, Universitat de Barcelona-Universitat Oberta de Catalunya.

Buxeda i Garrigós, J. (2008): "Revisiting the compositional data. Some fundamental questions and new prospects in Archaeometry and Archaeology", en J. Daunís-i-Estadella y J. Martín-Fernández (eds.), Proceedings of CODAWORK'08, The 3rd Compositional Data Analysis Workshop, May 27-30: 1-18. Girona, Universitat de Girona.

Buxeda i Garrigós, J.; Cau Ontiveros, M. A.; Gurt i Esparraguera, J. M. y Tuset i Bertran, F. (1995): “Análisis tradicional y análisis arqueométrico en el estudio de las cerámicas comunes de época romana", en Ceràmica comuna romana d'època alto-imperial a la Península Ibèrica. Estat de la qüestió, Monografies Emporitanes VIII: 39-60. Empúries, Conjunt Monument d'Empúries.

Buxeda i Garrigós, J. y Kilikoglou, V. (2003): “Total variation as a measure of variability in chemical data sets", en L. Van Zelst (ed.), Patterns and Process. A Festschrift in honor of Dr. Edward V. Sayre: 185-198. Suitland, Maryland, Smithsonian Center for Materials Research and Education.

Buxeda i Garrigós, J., Madrid i Fernández, M. e Iñañez, J.G. (2003): Estudio de caracterización arqueométrica de las producciones de cerámica vidriada de Talavera, Informe de Recerca FBG301873. Equip de Recerca Arqueomètrica de la Universitat de Barcelona. Barcelona, Universitat de Barcelona (inédito).

Buxeda i Garrigós, J., Madrid i Fernández, M., Iñañez, J. G. y Fernández de Marcos García, C. (2015): “Archaeometry of the Technological change in societies in contact. First examples for modern ceramics from the Crowns of Castile and Aragon”, en J. Buxeda i Garrigós, M. Madrid i Fernández y J. G. Iñañez (ed.), GlobalPottery 1. Historical Archaeology and Archaeometry for Societies in Contact, BAR International Series 2761: 3-25. Oxford, Archaeopress.

Buxeda i Garrigós, J., Mommsen, H. y Tsolakidou A. (2002): "Alterations of Na-, K-, and Rb- concentrations in Mycenaean pottery and a proposed explanation using X-ray diffraction". Archaeometry 44: 187-198.

Chaunu, P. (1983): Sevilla y América: siglos XVI y XVII. Sevilla, Publicaciones de la Universidad de Sevilla.

Deagan, K. A. (1987): Artifacts of the Spanish Colonies of Florida and the Caribbean, 1500-1800, vol. 1, Ceramics, Glassware, and Beads. Washington, Smithsonian Institution Press.

Egozcue, J.J., Pawlowsky-Glahn, V. (2011): "Basic concepts and procedures", en V. Pawlowsky-Glahn y A. Buccianti (ed), Compositional Data Analysis. Theory and Applications: 12-28. Chichester, Wiley. Elliott, J.H. (2005): La España imperial, 1469-1716. Barcelona, Vicens Vives. 
Ferrer, S.G., Buxeda i Garrigós, J., Iñañez, J.G., Amores Carredano, F. y Alzate Gallego, A. (2013): "Sevillian transport jars in early colonial America: the case of Santa María La Antigua del Darién (Colombia)". Open Journal of Archaeometry 1:e3: 10-15.

Ferrer, S. G., Buxeda i Garrigós, J., Iñañez, J. G. y Glascock, M. D. (2015a): "Local and European transport jars in Panama. Chemical and Mineralogical characterization", en J. Buxeda i Garrigós, M. Madrid i Fernández y J. G. Iñañez (ed.), GlobalPottery 1. Historical Archaeology and Archaeometry for Societies in Contact, BAR International Series 2761: 333-352. Oxford, Archaeopress.

Ferrer, S.G., Müller, N.S., Kilikoglou, V. (2015b): "High-performance transport jars for long-distance trading during the $16^{\text {th }}$ century", en J. Buxeda i Garrigós, M. Madrid i Fernández y J. G. Iñañez (ed.), GlobalPottery 1. Historical Archaeology and Archaeometry for Societies in Contact, BAR International Series 2761: 81-91. Oxford, Archaeopress.

Fournier, P. y Blackman, M. J. (2008): "Production, Exchange and Consumption of Glazed Wares in New Spain: Formation of a Database of Elemental Composition Through INAA". FAMSI. http:// www.famsi. org/reports/06014/06014Fournier.pdf [01/06/2014].

Goggin, J. M. (1968): Spanish Majolica in the New World: Types of the Sixteenth to Eighteenth Centuries. New Haven, Yale University Publications in Anthropology.

Hein, A., Müller, N.S., Day, P.M. y Kilikoglou, V. (2008): "Thermal conductivity of archaeological ceramics: The effect of inclusions, porosity and firing temperature". Thermochimica Acta 480: 35-42.

Hein, A., Müller, N. S. y Kilikoglou, V. (2009): “Great pots on fire: thermal properties of archaeological cooking ware", en K. T. Biró, V. Szilágyi y A. Kreiter (eds.), Vessels: inside and outside. Proceedings of the conference EMAC'07, 9th European meeting on ancient ceramics: 15-20. Budapest, Hungarian National Museum.

Hein, A., Tsolakidou, A., Iliopoulos, I., Mommsen, H., Buxeda i Garrigós, J., Montana, G. y Kilikoglou, V. (2002): "Standardisation of elemental analytical techniques applied to provenance studies of archaeological ceramics: an inter laboratory calibration study". The Analyst 127: 542-553.

Hughes, M.J. (2014): “Chemical Análisis of Pottery from the Tortugas Shipwreck (1622) by Plasma Spectrometry (ICPS)", en G. Stemm, S. Kingsley y E. Gerth, Oceans Odyssey 4. Pottery from the Tortugas Shipwreck, Straits of Florida: A Merchant
Vessel from Spain's 1622 Tierra Firme Fleet. Oxford, Oxbow Books.

Hunt Ortiz, M. A. (2001): "Excavación arqueológica del Castillo de San Jorge (Triana, Sevilla)". Anuario Arqueológico de Andalucía 1998, III-2: 811-823.

IGME (Instituto Geológico y Minero de España) (1975): Mapa Geológico de España E. 1:50.000, hoja 984, Sevilla [Material cartográfico]. Instituto Geológico y Minero de España, Servicio de Publicaciones del Ministerio de Industria, Madrid. 1 mapa y memoria. http://www.igme.es/internet/cartografia/cartografia/magna50.asp?hoja=984 [15/07/2014].

Iñañez, J.G. (2007): Caracterització arqueomètrica de la ceràmica vidrada decorada de la Baixa Edat Mitjana al Renaixement als centres productors de la Península Ibèrica. Tesis Doctoral, http://www.tdx. cat/handle/10803/2596. Barcelona, Universidad de Barcelona.

Jamieson, R. W. y Hancock, R. G. V. (2004): "Neutron activation analysis of colonial ceramics from Southern Highland Ecuador". Archaeometry 46: 569-583.

Lister, F. C. y Lister, R. H. (1974): "Maiolica in colonial Spanish America". Historical Archaeology 8(1): 17-52.

Lister, F. C. y Lister, R. H. (1982): Sixteenth Century Maiolica Pottery in the Valley of Mexico. Tucson, Department of Anthropology, University of Arizona.

Lister, F. C. y Lister, R. H. (1987): Andalusian Ceramics in Spain and New Spain. A Cultural Register from the Third Century B.C. to 1700. Tucson, University of Arizona Press.

Lorenzo Morilla, J., Vera Reina, M. y Escudero Cuesta, J. (1990): "Intervención arqueológica en c/ Pureza, núm. 44 de Sevilla". Anuario Arqueológico de Andalucía 1987. III. Actividades de Urgencia, Informes y Memorias: 574-580.

Maggetti, M. (1981): "Composition of roman pottery from Lousonna (Switzerland)", en M.J. Hughes (ed.), Scientific studies in ancient ceramics, British Museum Occasional Paper 19: 33-49. London, British Museum.

Maggetti, M. (1982): "Phase analysis and its significance for technology and origin", en J.S. Olin y A.D. Franklin (ed.), Archaeological Ceramics: 121-133. Washington D.C., Smithsonian Institution Press.

Maggetti, M. (1986): "Majolika aus Mexiko - ein archäeometrisches Fallbeispiel". Fortschritte der Mineralogie 64: 87 - 103.

Maggetti, M., Westley, H. y Olin, J. (1984): “Provenance and Technical studies of Mexican majolica using elemental and phase analysis", en J. B. 
Lambert (ed.), Archaeological Chemistry III, ACS Advances in Chemistry Series 205: 151-191. Washington D.C, American Chemical Society.

Maniatis, Y., Simopoulos, A. y Kostikas, A. (1981): "Moessbauer Study of the Effect of Calcium Content on Iron Oxide Transformations in Fired Clays". Journal of the American Ceramic Society 64: 263-269.

Martínez Shaw, C. (dir.) (1993): Sevilla, siglo XVI. El corazón en las riquezas del mundo. Madrid, Alianza Editorial.

Mena García, C. (1998): Sevilla y las flotas de Indias: la gran armada de Castilla del Oro (1513-1514). Sevilla, Universidad de Sevilla, Secretariado de Publicaciones.

Mercado Hervás, L.; Paz Jorva, M. J. y Mejías García, J. C. (2001): "Seguimiento arqueológico de la antigua estación de ferrocarril conocida como "Plaza de Armas", Sevilla". Anuario Arqueológico de Andalucía 1998. II. Actividades de Urgencia, Informes y Memorias: 715-721.

Morales Padrón, F. (1989): Historia de Sevilla. La ciudad del quinientos. Sevilla, Editorial Universidad de Sevilla.

Myers, J. E., Amores, F., Olin, J. y Pleguezuelo, A. (1992): "Compositional identification of Seville Majolica at Overseas sites”. Historical Archaeology 26: 131 - 147.

Ojeda Calvo, M. R. (1989): "Excavación arqueológica de apoyo a la restauración en el Real Monasterio de San Clemente de Sevilla". Anuario Arqueológico de Andalucía 1989. III Actividades de Urgencia, Informes y Memorias: 450-460.

Olin, J. S. y Blackman, M. J. (1989): “Compositional classification of Mexican majolica ceramics of the Spanish Colonial period", en R. O. Allen (ed.), Archaeological Chemistry IV, Advances in chemistry series 220, 4: 87-112. Washington DC, American Chemical Society.

Olin, J. S., Harbottle, G. y Sayre, E. V. (1978): "Elemental compositions of Spanish and Spanish-Colonial Majolica ceramics in the identification of provenience", en G. F. Carter (ed.), Archaeological Chemistry II, Advances in Chemistry Series, 171: 200-229. Washington DC, American Chemical Society.

Olin, J. S. y Myers, J. E. (1992): "Old and New World Spanish Majolica Technology”. Materials Research Society Bulletin 17: 32 - 38.

Picon, M. (1973): Introduction à l'étude technique des céramiques sigillées de Lezoux. Université Dijon, Centre de Recherches sur les techniques Gréco-romaines 2 .
Pleguezuelo, A. (1997): “Cerámica de Sevilla (12481841)", en T. Sánchez Pacheco (coord.), Summa Artis. Historia general del arte, vol. XLII: Cerámica española: 343-386. Madrid, Espasa Calpe.

Pleguezuelo, A. (2003a): "Centers of Traditional Spanish Mayólica”, en R. F. Gavin, D. Pierce y A. Pleguezuelo (ed.), Cerámica y Cultura. The Story of Spanish and Mexican Mayólica: 24-47. Albuquerque, University of New Mexico.

Pleguezuelo, A. (2003b): “Ceramics, business and economy”, en R. F. Gavin, D. Pierce y A. Pleguezuelo (ed.), Cerámica y Cultura. The Story of Spanish and Mexican Mayólica: 102-121. Albuquerque, University of New Mexico.

Polvorinos del Río, A. y Castaing, J. (2010): "Lustredecorated ceramics from a $15^{\text {th }}$ to $16^{\text {th }}$ century production is Seville". Archaeometry 52: 83-98.

R Core Team (2014): R: A language and environment for statistical computing. Viena, Austria, R Foundation for Statistical Computing <http://www.R-project.org/> [Consulta: 15 de diciembre de 2014].

Roberts, J. P. (1963): "Determination of the firing temperature of ancient ceramics by measurement of thermal expansion". Archaeometry 6: 21-25.

Sánchez Cortegana, J. M. (1994): El Oficio de ollero en Sevilla en el siglo XVI. Arte Hispalense 65. Sevilla, Diputación Provincial de Sevilla.

Sánchez, J. M. (1996): “La Cerámica Exportada a América en el Siglo XVI a Través de la Documentación del Archivo General de Indias. I. Materiales arquitectónicos y contenedores de mercancías". Laboratorio de Arte 9: 125-142.

Sánchez, J.M. (1998): “La Cerámica Exportada a América en el Siglo XVI a Través de la Documentación del Archivo General de Indias II: Ajuares Domésticos y Cerámica Cultural y Laboral". Laboratorio de Arte 11: 121-134.

Schwedt, A., Mommsen, H., Zacharias, N. y Buxeda i Garrigós, J. (2006): "Analcime crystallization and compositional profiles - comparing approaches to detect post-depositional alterations in archaeological pottery". Archaeometry 48: 237-251.

Tabales Rodríguez, M. A. (1991): “Investigación histórico-arqueológica en el Monasterio de San Clemente de Sevilla". Anuario Arqueológico de Andalucía 1991. III. Actividades de Urgencia, Informes y Memorias: 438-448.

Whitney, D. L. y Evans, B. W. (2010): “Abbreviations for names of rock-forming minerals". American Mineralogist 95: 185-187. 